\title{
Isolated massive stars in the Galactic center: The dynamic contribution from the Arches and Quintuplet star clusters
}

\author{
Maryam Habibi ${ }^{1,3}$, Andrea Stolte ${ }^{1}$, and Stefan Harfst ${ }^{2}$ \\ 1 Argelander Institut für Astronomie, Universität Bonn, Auf dem Hügel 71, 53121 Bonn, Germany \\ e-mail: [mhabibi; astolte] aastro.uni-bonn.de \\ 2 Technische Universität Berlin, Zentrum für Astronomie und Astrophysik, Hardenbergstraße 36, 10623 Berlin, Germany \\ e-mail: harfst@astro.physik.tu-berlin.de \\ 3 Member of the International Max Planck Research School (IMPRS) for Astronomy and Astrophysics at the Universities of Bonn \\ and Cologne, Germany
}

Received 11 November 2013 / Accepted 11 March 2014

\section{ABSTRACT}

\begin{abstract}
Recently, more than 100 Wolf-Rayet and OB stars were identified in the Galactic center. About a third of these sources are not spatially associated with any of the known star clusters in this region. We probe the distribution of drifted sources in numerical models of the massive clusters in the Galactic center and compare it to the observed distribution of isolated massive sources in this region. We find that stars as massive as $100 M_{\odot}$ drift away from the center of each cluster by up to $\sim 60 \mathrm{pc}$ using the cluster models. Our best model reproduces $\sim 60 \%$ of the known isolated massive stars out to $80 \mathrm{pc}$ from the center of the Arches cluster. This number increases to $70-80 \%$ when we only consider the region that is $\sim 20$ pc from the Arches cluster.
\end{abstract}

Key words. Galaxy: center - open clusters and associations: individual: Arches - stars: early-type - stars: Wolf-Rayet open clusters and associations: individual: Quintuplet - methods: numerical

\section{Introduction}

Galactic nuclei are ideal laboratories to investigate star formation in extreme conditions, such as in a strong tidal field, high UV radiation, and a strong magnetic field. The only galactic nucleus where we can resolve the stellar population into individual stars is the center of our Galaxy at a distance of $\sim 8.0 \mathrm{kpc}$ (Ghez et al. 2008; Gillessen et al. 2009). However, the conditions for star formation and the dynamics of this region have yet to be understood as it harbors dense molecular clouds, a high star formation rate per unit volume, and the largest concentration of massive stars and star clusters in the Milky Way (e.g., Morris \& Serabyn 1996; Figer \& Morris 2002; Ferrière et al. 2007).

The Galactic center (GC) region hosts three starburst clusters with masses in excess of $\sim 10^{4} M_{\odot}$ and core radii of $\sim 0.15-1 \mathrm{pc}$ (Eckart et al. 1993; Figer et al. 2002; Espinoza et al. 2009). These three compact and massive clusters are the young nuclear cluster surrounding the supermassive black hole, the Quintuplet cluster, and the Arches cluster. Recent observations of isolated sources in the GC region revealed that the field stars in this area, similar to these three clusters, encompass many massive sources (Dong et al. 2011a; Mauerhan et al. 2010a; Mauerhan et al. 2010b; Wang et al. 2010). A population of distributed, very massive Wolf-Rayet (WR) stars with initial masses in excess of 20-40 $M_{\odot}$ were detected within a few dozen of pc from the super-massive black hole, Sgr A*, by X-ray observations, which are accompanied by spectroscopic studies and Paschen- $\alpha(\operatorname{Pa} \alpha)$ narrow-band imaging with Hubble Space Telescope (HST, Wang et al. 2010; Dong et al. 2011a). Up to now, more than $100 \mathrm{WR}$ stars and $\mathrm{O}$ supergiants have been spectroscopically identified in the Galactic center region (Mauerhan et al. 2010a), including the known cluster members.
As about a third of these sources are located outside of the three massive starburst clusters, it has been suggested that they provide evidence for isolated high-mass star formation in the GC (Dong et al. 2011b; Oskinova et al. 2013). Observations of massive stars in the solar neighborhood show that generally massive stars form in groups and associations (Lada \& Lada 2003; Zinnecker \& Yorke 2007; Gvaramadze \& Bomans 2008), but it is not clear if we can generalize these findings to different galactic environments. On the other hand, dynamical evolution of stellar populations in the GC region can become dramatic under the strong effect of the GC tidal field. If so, dense and massive clusters like the Arches and Quintuplet can shape the distribution of the field stars in the region.

The Arches and Quintuplet clusters have been observed to be already mass segregated at ages of 2-6 Myr. A recent study by Hußmann et al. (2012) showed that the Quintuplet cluster at an age of 3-5 Myr exhibits a flat mass function slope of $-1.68 \pm 0.1$ in the cluster center compared to the standard Salpeter (1955) initial mass function (IMF) of -2.3 . A similarly flat mass function was found in the central region of the Arches cluster, but the slope increases substantially toward larger radii (Figer et al. 1999a; Stolte et al. 2005; Espinoza et al. 2009; Habibi et al. 2013). A study by Harfst et al. (2010) implemented $\mathrm{N}$-body simulations of the Arches cluster to investigate the internal dynamical evolution of the cluster. By comparing their models to the observational data from the central $0.4 \mathrm{pc}$ of the Arches cluster, they could constrain the initial conditions and construct a dynamical model of the Arches cluster that best represented the central stellar mass distribution, hereafter the bestfitting model of the Arches cluster. From this model, a steep increase of the stellar mass function slope as a function of the cluster center distance was predicted. The Arches cluster was later studied by Habibi et al. (2013) to a larger radius of $\sim 1.5 \mathrm{pc}$. 
Observations of the high-mass part of the mass spectrum in this study $\left(M \gtrsim 10 M_{\odot}\right)$ revealed a depletion of massive stars in the cluster outskirts. In this previous study, we compared the measured slope of the mass function to the slopes predicted by the $N$ body simulations in different annuli out to the tidal radius of the Arches cluster. This comparison showed that the Arches cluster exhibits characteristics of a normal, which is defined as a cluster with a normal initial mass function, but dynamically evolved cluster. The dynamical evolution of the Arches and Quintuplet clusters, however, not only changes the distribution of stars inside the cluster, but also changes the distribution of field stars in the GC region. Through gravitational interactions between stars in dense, compact clusters, stars can accelerate to become runaways (e.g., Poveda et al. 1967; Gies \& Bolton 1986). Moreover, the dynamical evolution of clusters under the influence of the Galactic tidal field leads to the formation of tidal arms. These tidal structures are mostly observed for globular clusters, which evolve for many Gyrs (e.g., Odenkirchen et al. 2001). As massive clusters in the GC dissolve within a few Myrs (Kim et al. 2000; Portegies Zwart et al. 2001a), dynamical evolution under the effects of the strong tidal field of the GC leads to the formation of extended tidal structures during shorter timescales. These tidal structures, in turn, can significantly contribute to the field stars in the GC region.

In this study, we analyze the best-fitting Arches model presented by Harfst et al. (2010), which is extended to incorporate the effect of the Galactic center tidal field, to investigate the contribution of the Arches and Quintuplet clusters to the observed population of isolated massive stars detected by Mauerhan et al. (2010a). This paper is organized as follows: in Sect. 2, a summary of observational studies to detect massive stars in the GC region with our criteria to construct an observational reference sample is presented. In Sect. 3, we describe the computational methods, a grid of different models based on distinct physical assumptions, and a method to find the best-matching model to reproduce the observations. In Sect. 4, we analyze this bestmatching model to derive the spatial distribution of the massive drifted sources compared to the observed population. The velocity distribution of the drifted sources from the modeled clusters and their expected spatial and mass distributions are also predicted in this section. A summary of our findings is presented in Sect. 5 .

\section{The observed population of Wolf-Rayet stars and $O$ supergiants}

A recent HST/NICMOS Pa $\alpha$ survey of the Galactic center was carried out by Wang et al. (2010) to study the distribution of young massive stars in the GC region. The survey covered the central region of the Galaxy, $\approx 0.65^{\circ} \times 0.25^{\circ}(l, b)$. At the distance of the GC from the Sun $(8 \mathrm{Kpc}$; Ghez et al. 2008 , Gillessen et al. 2009), an angular distance of $1^{\circ}$ corresponds to a projected distance of $\approx 140 \mathrm{pc}$, such that the survey covers an area of $91 \times 35 \mathrm{pc}^{2}$. Wang et al. (2010) employed two narrow band filters, F187 $(1.87 \mu \mathrm{m})$ and F190 $(1.90 \mu \mathrm{m})$, to detect sources with $\operatorname{Pa} \alpha$ excess. The $\operatorname{Pa} \alpha$ emission line $(1.87 \mu \mathrm{m})$ is the strongest line in the infrared band, which mainly originates in warm and hot ionized gas. Accordingly, $\mathrm{Pa} \alpha$ point sources are chiefly produced by evolved massive stars (Mauerhan et al. 2010a). Later, Mauerhan et al. (2010a) performed a follow-up near-infrared spectroscopic survey targeting the strongest $\mathrm{Pa} \alpha$ sources with bright $K_{\mathrm{s}}$-band counterparts $\left(K_{\mathrm{s}}<12.7 \mathrm{mag}\right)$. For this reason, Mauerhan et al. (2010a) employed the $J H K_{\mathrm{s}}$ photometry down to $15.6 \mathrm{mag}$, which is taken from the 2MASS and SIRIUS catalogs (2MASS: Two Micron All Sky Survey; Cutri et al. 2003, SIRIUS: Simultaneous three-color InfraRed Imager for Unbiased Surveys; Nagayama et al. 2003). These magnitude ranges cover the predicted magnitude for WR stars in the GC region, which is dominated by WNL (late nitrogen-rich WR) stars $(K<12$; Figer 1995). Fainter WNE (early nitrogenrich WR) stars are still detectable due to their strong Pa $\alpha$ emission. Therefore, their sample of WN (nitrogen-rich WR) stars in the $\mathrm{Pa} \alpha$ survey area is nearly complete, whereas the provided sample of WC (carbon-rich WR) stars is presumably not as complete (see Sect. 6.1 of Mauerhan et al. 2010a for a detailed discussion).

In this study, we use the catalog of isolated massive sources in the GC that is provided by Mauerhan et al. (2010a) to perform a statistical analysis. Mauerhan et al. (2010a) present a catalog of all the identified evolved massive stars in the GC, including their newly discovered sources. This includes those previously identified in the Arches, Quintuplet and the Nuclear star clusters, as well as those found outside the $\mathrm{Pa} \alpha$ survey coverage. In the presented catalog by Mauerhan et al. (2010a), sources that lie outside the $\mathrm{Pa} \alpha$ survey area (located at galactic longitudes of $\left.0.2^{\circ} \lesssim l \lesssim 0.6^{\circ}\right)$ are taken from the study by Mauerhan et al. (2010b).

Mauerhan et al. (2010b) performed a spectroscopic study on a pre-compiled list of potential near-infrared counterparts to hard $\mathrm{X}$-ray sources. They derived the candidate list by matching the Chandra catalog of X-ray sources in the central $2^{\circ} \times 0.8^{\circ}(l, b)$ of the galaxy (Muno et al. 2009) to the available near-infrared surveys in the region. However, the studied sample by Mauerhan et al. (2010b) in the region outside the $\mathrm{Pa} \alpha$ survey area suffers from selection bias. For the spectroscopic follow-up, Mauerhan et al. (2010b) give precedence to the sources close to previously observed diffuse mid-infrared structures. Diffuse infrared emission is the characteristic of young star-forming regions in the GC. Mauerhan et al. (2010b) mostly studied these regions which may harbor ongoing star formation with the hope to enhance the detection rate of massive stars. To avoid the selection bias, we limit our sample to the region that is covered by the $\operatorname{Pa} \alpha$ survey by selecting sources at longitudes $l<0.23^{\circ}$ and latitudes $b>-0.1^{\circ}$ from their catalog (see Fig. 3). We utilize this truncated list of observed isolated massive sources as our observational reference sample ${ }^{1}$. The final employed list of 35 observed isolated massive stars in the GC region is presented with positions and spectral types in Table 2, along with their reference studies. This table contains $13 \mathrm{WN}$ stars, $11 \mathrm{WC}$ stars, $1 \mathrm{LBV}$ star, and $10 \mathrm{OB}$ supergiants outside the three clusters in the $\mathrm{Pa} \alpha$ survey region (see Fig. 3).

\section{Dynamical cluster model}

\section{1. $N$-body simulations}

The dynamical cluster models presented here are based on the work by Harfst et al. (2010), who compared the results of numerical simulations with the observational data from the central $0.4 \mathrm{pc}$ of the Arches cluster in detail (Stolte et al. 2005). They constrained the initial conditions and found a best-fitting Arches

\footnotetext{
1 A more recent study by Dong et al. (2012) includes fainter massive field stars in the GC ( $\left.K_{\mathrm{s}} \gtrsim 13 \mathrm{mag}\right)$. Their catalog is not employed in our study, as most of these stars still do not have available spectroscopic identifications. Moreover, as they have not performed a spectroscopic survey, the spatial distribution of sources with previous spectroscopic identifications is probably biased to the target area of earlier studies.
} 
model. This best-fitting Arches model is used here as a starting point for the simulations.

The setup of the dynamical models of the Arches cluster, as described in the following, is similar to the models presented by Olczak et al. (2012). For our simulations, the age of the Arches cluster is set to $t_{\text {age }}=2.5 \mathrm{Myr}$ (Blum et al. 2001; Najarro et al. 2004; Martins et al. 2008), and we assume that the cluster is initially (at $t=0 \mathrm{Myr}$ ) gas-free and in virial equilibrium and that its mass is distributed by following a King profile (King 1966). A single-aged stellar population is used, and no initial mass segregation is taken into account.

According to the best-fit model of Harfst et al. (2010), the observed present-day mass function of the Arches cluster is the result of a dynamically evolved Salpeter IMF (Salpeter 1955) at the given age of the cluster. The total mass of the cluster is then given by the total number of massive stars (stars with $m>10 M_{\odot}$ which are complete in the observational data) and the lower mass limit of the IMF. For the latter, we adopt $m_{\text {low }}=0.5 M_{\odot}$. As a result, the total mass of the cluster model is $M=4.8 \times 10^{4} M_{\odot}$, which corresponds to an initial number of massive stars of $N_{m>10} M_{\odot} \approx 500$.

The King profile concentration parameter was set to $W_{0}=3$. The initial size of the cluster is given by the initial virial radius, which was set to $0.77 \mathrm{pc}$ (see Harfst et al. 2010). The corresponding core radius is about $0.4 \mathrm{pc}$ initially, and shrinks down to the observed $0.2 \mathrm{pc}$ due to the dynamical evolution of the cluster (Espinoza et al. 2009).

Stellar evolution and the orbit of the Arches cluster in the Galactic center potential have been neglected in the best-fit model of Harfst et al. (2010). Here, we extend that model, as described below, to include these two effects. The simulations were carried out with the direct $N$-body code kira from the starlab-package (McMillan 1996; Portegies Zwart et al. 2001b; Hut 2003), which includes modules for stellar evolution and an external potential. The stellar evolution module includes mass loss by stellar winds and binary evolution (Eggleton et al. 1989; Portegies Zwart \& Verbunt 1996; Tout et al. 1997; Langer 1998). The external potential that was used in our simulations is that of a power-law mass distribution with $M_{\mathrm{gal}}(r)=$ $4.25 \times 10^{6}(r / \mathrm{pc})^{1.2} M_{\odot}$ and is based on Mezger et al. (1999).

The cluster orbit in the potential is given by the six phase space coordinates composed of the $3 \mathrm{D}$ position and velocity of the cluster at any given time. For the present day $(t=2.5 \mathrm{Myr})$, we know the line-of-sight velocity $\left(95 \mathrm{~km} \mathrm{~s}^{-1}\right.$, Figer et al. 2002) and the projected position ( $26 \mathrm{pc}$ from the Galactic center). Additionally, Stolte et al. (2008b) determined the proper motion of the Arches cluster and found a 2D velocity of $212 \mathrm{~km} \mathrm{~s}^{-1}$ (anti-)parallel to the Galactic plane. The proper motion of the cluster was later revised to a slightly lower value of $172 \pm$ $15 \mathrm{kms}^{-1}$ (see Clarkson et al. 2012 for details). We have therefore adopted the mean present-day 2D velocity of $190 \mathrm{kms}^{-1}$ for our cluster simulations. As the difference in the 3D space motion is small, we do not expect the orbit to change substantially. If we define a coordinate system in which the $x$-axis is along the Galactic plane, the $y$-axis along the line-of-sight, and the $z$-axis toward the Galactic north pole, we obtain the following vectors for the position, $\boldsymbol{r}_{\text {cluster }}$, and velocity, $\boldsymbol{v}_{\text {cluster }}$, of the cluster at the present epoch:

$\boldsymbol{r}_{\text {cluster }}=\left(-24, R_{\mathrm{GC}}+d_{\mathrm{los}}, 10\right) \mathrm{pc}$

$\boldsymbol{v}_{\text {cluster }}=(-190,95,0) \mathrm{km} \mathrm{s}^{-1}$,

where the not well-known $d_{\mathrm{los}}$ determines the distance of the cluster to the Galactic center along the line of sight.
Stolte et al. (2008a) discussed the formation of the Arches cluster inside the central $200 \mathrm{pc}$ of the Galaxy as one of the most likely formation scenarios. Furthermore, considering its orbital properties and the measured foreground extinction of the cluster, which is lower than the extinction found toward the central parsec, Stolte et al. (2008a) concluded that the cluster is likely in front of the GC today. For the simulations, we therefore assume a line-of-sight location of $d_{\mathrm{los}}=-100 \mathrm{pc}$ in front of the $\mathrm{GC}$ at its present age of $2.5 \mathrm{Myr}$.

The present-day position and velocity can be used to numerically integerate the orbit backwards in time to find the initial position and velocity at $t=0 \mathrm{Myr}$. From there, the full cluster was integrated in kira until $t=6 \mathrm{Myr}$, which includes the effects of stellar evolution and the GC tidal field. A total of ten random realizations of this model were integrated to allow for statistical analysis. A full snapshot of the cluster (mass, position, and velocity for every star) was stored every $\sim 0.5$ Myr. From these snapshots, the projection on the plane of the sky is easily obtained.

\subsection{Model grid}

The Arches and Quintuplet clusters share similar characteristics. Other than their proximity, observations yield similar estimates of the present-day mass of the clusters (Hußmann 2014; Habibi et al. 2013; Clarkson et al. 2012). Additionally, preliminary results on the proper motion study of the Quintuplet cluster suggests that the motion of the Quintuplet cluster is similar to the orbital motion of the Arches cluster (Stolte et al. 2011). However, the Quintuplet is slightly older than the Arches (2-3 Myr vs. 3-5 Myr, Najarro et al. 2004 and Figer et al. 1999b, respectively) and appears to be more dispersed than the Arches cluster. Quintuplet is more than 100 times less dense than the Arches cluster (Figer 2008). These properties are suggestive to consider the Quintuplet cluster as an older representation of the Arches cluster.

To model the observed population of massive field stars in the GC region, we need to consider the contribution of both the Arches and the Quintuplet clusters. Our simulated cluster provides the contribution from the Arches cluster. We assume the Quintuplet cluster is a snapshot of the Arches cluster at older ages as it evolves in the GC region. To construct a single model that contains both the Arches and Quintuplet clusters, the data from the two simulated clusters are combined, so that their projected distance on the plane of the sky matches the observations. Assuming the GC distance of $8 \mathrm{kpc}$, the observed separation of the clusters along the Galactic plane and the Galactic north pole is $\sim 6 \mathrm{pc}$ and $\sim 11 \mathrm{pc}$, respectively.

Our models do not include the Nuclear cluster, since we do not expect that ejected sources from the Nuclear cluster linger in the GC region. Schödel et al. (2009) observed the proper motions of stars within a distance of $1.0 \mathrm{pc}$ from Sgr A*. They found few stars with proper motion velocities, which exceed $400 \mathrm{~km} \mathrm{~s}^{-1}$ in both the radial and tangential axes. Out of these candidates only one source has a velocity uncertainty below $1 \sigma$. It is noteworthy that the black hole governs the dynamics close to the center of the Nuclear cluster. However, as first pointed out by Hills (1988), mechanisms like interaction of a massive binary with the black hole produce ejectors with hyper-velocities of $\gtrsim 1000 \mathrm{~km} \mathrm{~s}^{-1}$. These hyper-velocity ejecta traverse our target region of $50 \mathrm{pc}$ around the GC in $\sim 10^{4}$ yrs. Comparing this value to the maximum predicted ejection rate of $10^{-4} \mathrm{yr}^{-1}$ (Yu \& Tremaine 2003; Perets et al. 2007), we expect $\sim 1$ hyper-velocity star originated 
Table 1. Calculated histogram difference, $H_{\mathrm{d}}$, between the observed isolated massive sources by Mauerhan et al. (2010a) and different models.

\begin{tabular}{ccccccc}
\hline \hline \multicolumn{7}{c}{$H_{\mathrm{d}}$} \\
\hline & WR-L $=20 M_{\odot}$ & StdDev & WR-L $=40 M_{\odot}$ & StdDev & WR-L $=60 M_{\odot}$ & StdDev \\
\hline$Q_{\text {age }}=4 \mathrm{Myr}$ & 17.4 & $\sigma_{4,20}=4.38$ & 4.48 & $\sigma_{4,40}=1.43$ & 6.38 & $\sigma_{4,60}=1.03$ \\
$Q_{\text {age }}=4.5 \mathrm{Myr}$ & 26.55 & $\sigma_{4.5,20}=6.93$ & 5.96 & $\sigma_{4.5,40}=1.15$ & 5.63 & $\sigma_{4.5,60}=0.88$ \\
$Q_{\text {age }}=5.0 \mathrm{Myr}$ & 11.69 & $\sigma_{5,20}=1.79$ & $\mathbf{3 . 7 8 4}$ & $\sigma_{5,40}=0.75$ & 7.25 & $\sigma_{5,60}=0.81$ \\
\hline
\end{tabular}

Notes. Compared models assume an age of 4, 4.5, and 5 Myr for the Quintuplet cluster and 2.5 Myr for the Arches. The initial mass for the massive stars is considered to be 20,40, and $60 M_{\odot}$ in these models. The model, which assumes an age of 5 Myr for the Quintuplet cluster and an initial mass of $40 M_{\odot}$ for WR progenitors, is found to have the lowest $H_{\mathrm{d}}$ and is the most similar model to the observed distribution. The standard deviations for the $H_{\mathrm{d}}$ values are derived by individual comparison of the ten random realizations of each model.

in the Nuclear cluster to contribute to the observed sample of massive stars in the GC at any given time.

To obtain an estimate of the number of drifted sources ${ }^{2}$, which are expected to originate in the Quintuplet, we evolve the model for a longer time. The age of the Arches cluster is estimated to be 2.5 Myr. The age of the Quintuplet cluster, however, is less constrained. Figer et al. (1999b) conducted a photometric and spectroscopic study on massive stars in the cluster and derived an age of 3 to $5 \mathrm{Myr}$ based on the types of the stars they found. A more recent spectral analysis of WN stars by Liermann et al. (2010) favors a younger age of 3 million years, while OB stars in the cluster populate a 4 Myr isochrone on the HR diagram (Liermann et al. 2012). To account for the age uncertainty of the Quintuplet cluster, we use three different snapshots of our model at 4, 4.5, and $5 \mathrm{Myr}$ to represent the current extended population originating from the Quintuplet. Each of the three snapshots are combined with the Arches model at $2.5 \mathrm{Myr}$ to estimate the total number of isolated massive stars originating from both clusters in the GC today.

Another parameter, which has a significant effect on the predicted number of WR stars in the region, is the minimum initial mass of a WR progenitor star. A WR star represents a massive star at its late evolutionary stage. Eldridge \& Tout (2004) concluded that the minimum initial mass of a WR progenitor is $\sim 25 M_{\odot}$ at solar metallicity. However, modeling WR stars is still a topic of active research. Recent Geneva stellar evolution tracks (Ekström et al. 2012) determine a minimum initial mass of $32 M_{\odot}$ for a He-burning star with an age of 5.26 Myr. In these models, the minimum initial mass of a $4.97 \mathrm{Myr}$ star, which is at its core He-exhaustion phase, is $40 M_{\odot}$. For rotating models, these numbers are higher and are close to $60 M_{\odot}$. Since observed WRs are defined by their prominent broad emission lines, the spectral analysis of WR stars is only possible through detailed model atmospheres. Hamann et al. (2006) performed a spectral analysis on a large sample of Galactic WN stars using the Potsdam Wolf-Rayet (PoWR) model atmosphere code. Based on the rough qualitative agreement of their analysis and available non-rotating Geneva tracks at the time (Meynet \& Maeder 2003 ), they found a minimum initial mass of $37 M_{\odot}$ for a star to reach any WR phase. Later, Sander \& Hamann (2012) studied Galactic WC stars using the same model atmosphere code. They conclude that these stars are evolved from progenitors of 20 to $45 M_{\odot}$. On the basis of current knowledge of evolved massive stars, we consider three different minimum initial masses of 20 , 40 , and $60 M_{\odot}$ for a star to eventually evolve into a WR star.

\footnotetext{
2 We consider sources outside the tidal radius $(\sim 1.6 \mathrm{pc}$; Habibi et al. 2013) of the Arches and Quintuplet clusters (Hußmann 2014) that are part of the tidal arms as drifted sources.
}

Based on these different assumptions about the age of the Quintuplet cluster and the initial mass of the WR progenitors, we construct nine models of massive stars in the Arches and Quintuplet clusters. We compare the population of drifted massive stars of the nine models with the observed isolated massive sources (see Table 1).

\subsection{Comparison of the observed distribution of isolated high-mass stars with the model grids}

\subsubsection{The concept of the histogram difference}

By constructing a grid of different models (see Sect. 3.2), we are interested in finding the most similar model to the observed population. To measure the deviation of the models from the observed data, histograms of the data and each model are constructed by calculating spatial distances of all the stars, including the Quintuplet members, to the center of the Arches cluster as a reference point. One way to quantify histogram differences is to use the number count difference in each bin, which is equivalent to calculating the classic Euclidean distance between the feature vectors $^{3}$ of two distributions. The square of the classic Euclidean distance of two $N$-dimensional vectors $\boldsymbol{P}$ and $\boldsymbol{Q}$ is defined as follows:

$D_{\text {euclid }}^{2}(\boldsymbol{P}, \boldsymbol{Q})=(\boldsymbol{P}-\boldsymbol{Q}) \cdot(\boldsymbol{P}-\boldsymbol{Q})^{\mathrm{T}}=\sum_{i=1}^{N}\left(p_{i}-q_{i}\right)^{2}$.

Consider the three different sample spatial distributions illustrated in Fig. 1, which are comparable representations of a background distribution with two toy-clusters with different cluster distances. The histograms of their distributions along the $x$-axis show peaks with similar height. Intuitively, we appraise the first two distributions to be more similar compared to the third. However, comparing the Euclidean distance between the feature vectors of each pair shows the same level of similarity with just one of the major peaks shifted to another radial bin. The Euclidean distance assumes there is no relationship between individual components (number counts in each bin here) and does not reflect the similarity of the $i$ th bin of the feature vector $\boldsymbol{P}$ to the $(i+1)$ th neighboring bin of the compared feature vector $\boldsymbol{Q}$. Therefore, the simple Euclidean distance, which is the difference of the count in each bin, is not appropriate for distributions, which exhibit correlations of the feature vector components. For example, more fully occupied bins might compensate less populated neighboring bins.

\footnotetext{
3 A feature vector is a vector for which the components of the vector represent properties of some data set. Here, constructed histograms represent the feature vectors of each distribution.
} 

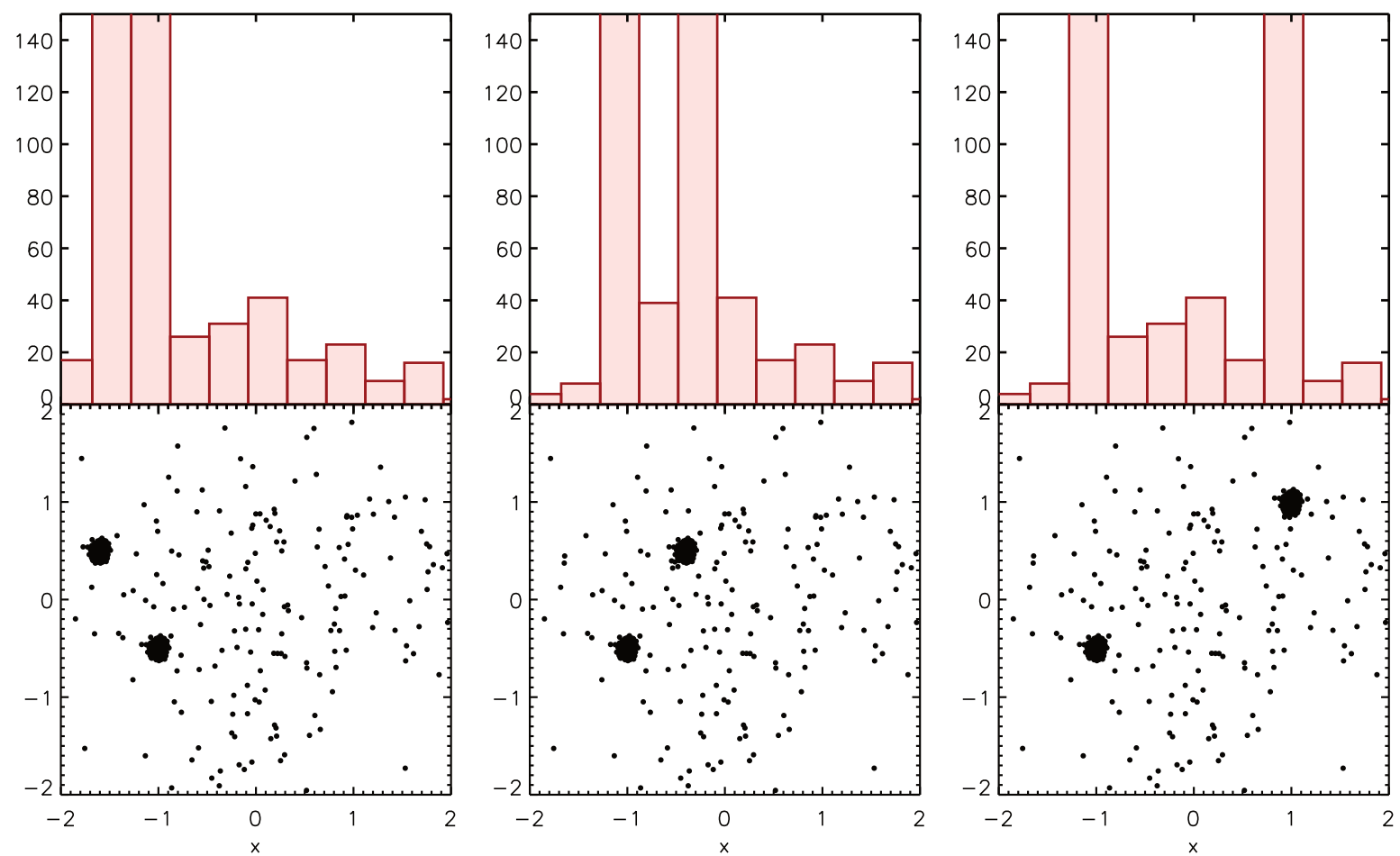

Fig. 1. Three different artificial distributions are shown as examples, accompanied by the histograms of their distributions along the $x$-axis. The background population along with one of the over-densities are similar in the three plots.

To overcome such a problem, Hafner et al. (1995) suggested a method for retrieval of images based on their color histograms using a quadratic distance measure. Assuming the $N$-dimensional distributions $\boldsymbol{P}$ and $\boldsymbol{Q}$, if $\boldsymbol{F}=\boldsymbol{P}-\boldsymbol{Q}$, the quadratic distance can be presented as: $D_{Q}^{2}(\boldsymbol{P}, \boldsymbol{Q})=\boldsymbol{F}^{\mathrm{T}} \mathbf{A} \boldsymbol{F}$. The matrix $A=\left[a_{i j}\right]$ is a weighting matrix, which allows for individual weighting of the cross-correlation between the bins $i$ and $j$. Therefore, we can calculate the histogram difference, $H_{\mathrm{d}}$, by cross-matching between different components:

$H_{\mathrm{d}}=D_{\text {hist }}^{2}(\boldsymbol{P}, \boldsymbol{Q})=\boldsymbol{F}^{\mathrm{T}} \mathbf{A} \boldsymbol{F}=\sum_{i, j}^{N}\left(p_{i}-q_{i}\right)\left(p_{j}-q_{j}\right) a_{i j}$.

The histogram difference as a form of quadratic distance is successfully applied to compare color histograms of different multimedia databases (e.g., Ankerst et al. 1998). Here, we adopt the idea presented by Hafner et al. (1995) to calculate the histogram difference, $H_{\mathrm{d}}$, between various models and the observed data. Our applied weighting matrix is constructed as:

$a_{i j}=\exp \left(-\sigma d_{i j}^{2}\right)$

where $d_{i j}$ is the Euclidean distance between bins $i$ and $j$ and $\sigma$ is a constant which, determines the shape of the weighting matrix (see Hafner et al. 1995 for details). This method allows us to consider the influence of neighboring bins on each other. Hence, the weighting matrix should increase the effect of cross-correlations between bins $i$ and $j$ as a function of distance between the two bins. This objective is fulfilled by choosing lower $\sigma$ values to increase the non-diagonal entries of the weighting matrix, $A$. On the other hand, the weighting matrix $A=\left[a_{i j}\right]$ should generate a quadratic distance, $H_{\mathrm{d}}$, which is nonnegative. This requirement demands for higher $\sigma$ values. In our analysis, the positive semidefinite weighting matrix with relatively large non-diagonal entries is achieved with a $\sigma$ value of 12 .
The defined histogram difference, $H_{\mathrm{d}}$, varies between 0 and $\infty$. Analogous to the Euclidean distance, the $H_{\mathrm{d}}$ value cannot be used to define the absolute "closeness" or in particular "similarity" in this context. Nevertheless, we can use it to compare distinct models and to find the most similar model to our observed reference data set by determining the relative "similarity".

\subsubsection{Selection of the best-matching model}

We compute the histogram difference, $H_{\mathrm{d}}$, between the observed population of isolated massive stars in the GC and different models (see Sect. 3.2) with the aim of selecting the best-matching model. Table 1 lists models with different assumptions on the age of the Quintuplet cluster and on the initial masses of stars that are currently observed as WN, WC, or OB stars. The final feature vector of the spatial distribution for massive stars in each constructed model of both the Arches and Quintuplet clusters is compared to the observed data in the GC. Feature vectors of the models are constructed as histograms for the spatial distances of all the stars in the two clusters with reference to the center of the Arches cluster and the average of ten random realizations for each model. The feature vector of the observed population is likewise constructed as the histogram that contains the number of observed high-mass stars with respect to the center of the Arches cluster. To estimate the uncertainty in the histogram distance between the average model and the observations, the ten random realizations are also individually compared to the observed distribution. The calculated standard deviation of the resulting $H_{\mathrm{d}}$ values are reported for each model in Table 1 . Since we are only interested in the population of drifted or ejected stars, sources that lie inside the tidal radius $(\sim 1.6 \mathrm{pc}$; Habibi et al. 2013) of the Arches, Quintuplet, and Nuclear clusters are excluded from the data and the models. The bin size is chosen to be $3 \mathrm{pc}$ to avoid random fluctuations. Our experiment shows that the final calculated $H_{\mathrm{d}}$ value is not very sensitive to 


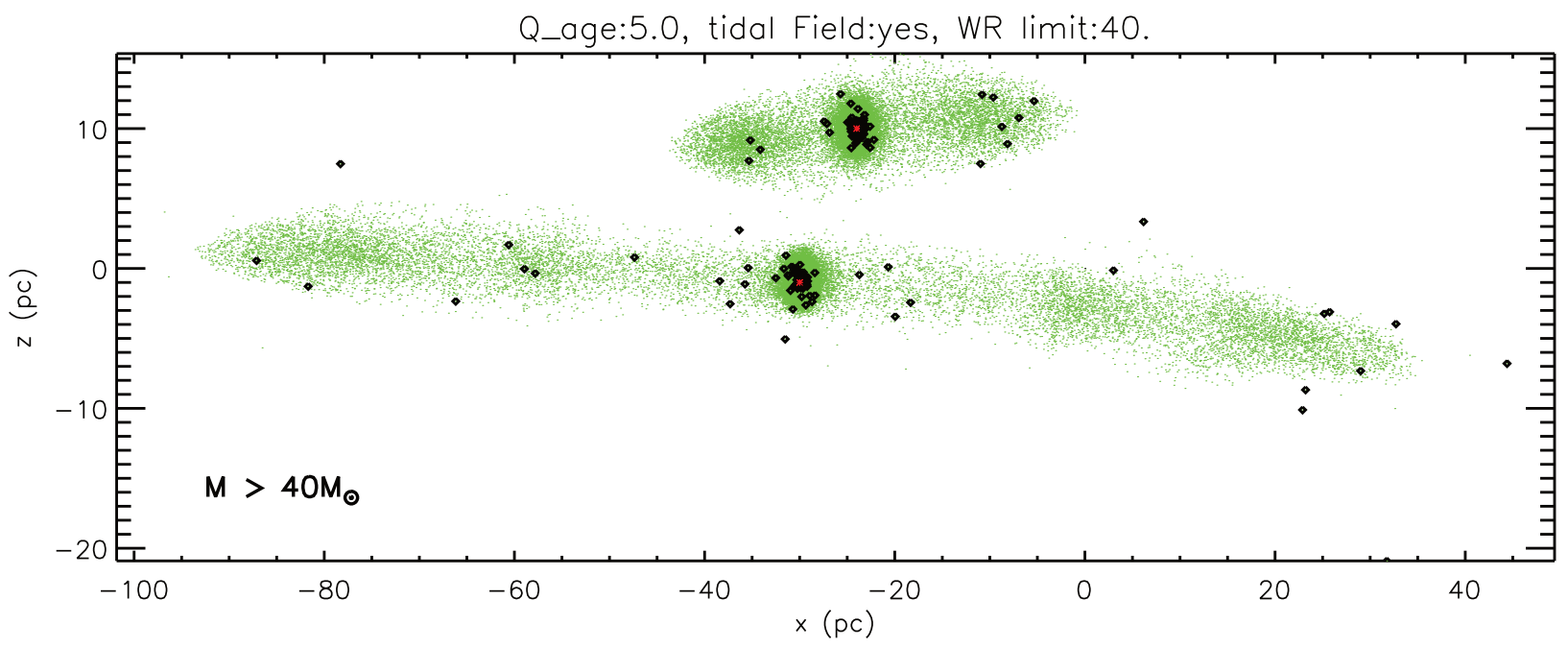

Fig. 2. One realization of the best-matching model is projected on the plane of the sky. Green dots represent the cluster members. Sources with initial masses exceeding $40 M_{\odot}$ are illustrated by filled diamonds. The $x$-axis is along the Galactic plane, and the $z$-axis is toward the Galactic north pole.

the bin size, since our method accounts for the impact of neighboring bins on each particular bin. The model with an age of 5 Myr for the Quintuplet cluster and an initial mass of $40 M_{\odot}$ for massive stars is found to have the lowest $H_{\mathrm{d}}$ value and is the most similar model to the observed distribution. We use this best-matching model for the comparison to the observations and further analysis.

\section{Results}

The Arches cluster moves rapidly through the high-density environment in the GC (e.g., Stolte et al. 2008b). As the cluster moves along its orbit, two-body relaxation in the cluster accelerates some stars to exceed the escape velocity, which is variable and determined by the tidal force. These stars either take over the cluster center or fall behind depending on their differential velocities. This known phenomenon populates the two extended tidal arms. In this section, we present the comparison of the massive stars outside the Arches and Quintuplet clusters in the best-matching model, which mostly belongs to the tidal arms, with the observed massive sources in isolation (Mauerhan et al. 2010a). In Sect. 4.1, simulations are compared to the data in terms of the number of isolated sources and their spatial distribution. In Sect. 4.4, the velocity variation along the tidal structures are analyzed to obtain a general picture of dynamical evolution of the cluster as it moves along its orbit.

\subsection{The spatial distribution of drifted and observed high-mass stars}

The best-matching model of massive sources outside the Arches and Quintuplet clusters assumes an age of $5 \mathrm{Myr}$ for the Quintuplet cluster and $M>40 M_{\odot}$ for WR progenitors. Figure 2 demonstrates the projection of one of the realizations of the best-matching model on the plane of the sky. In this model, each tidal arm of the Arches cluster extends out to $20 \mathrm{pc}$ along the Galactic plane, whereas tidal arms of the Quintuplet cluster stretch out to $65 \mathrm{pc}$. Massive stars in both clusters, $M>40 M_{\odot}$, are mostly concentrated around the cluster center. Mass segregation of the Arches cluster has been observationally confirmed, since the observed slope of the mass function increases with radius (Espinoza et al. 2009; Habibi et al. 2013). Observations of the high-mass part of the mass spectrum of the Arches cluster $\left(M \gtrsim 10 M_{\odot}\right.$ ) out to its tidal radius revealed a depletion of massive stars in the cluster outskirts (Habibi et al. 2013). In our previous study, we derived a present-day mass function slope of $-3.21 \pm 0.30$ in the outer annulus $(0.4<r<1.5 \mathrm{pc})$ as compared to a flat slope of $-1.50 \pm 0.35$ in the core $(r<0.2 \mathrm{pc})$, where the Salpeter slope is -2.3 . The Quintuplet cluster exhibits a density that is about 100 times lower in the core compared to the Arches cluster (Figer 2008). The present-day mass function of the Quintuplet cluster is also characterized by a flat slope, $-1.68 \pm 0.1$ (Hußmann et al. 2012). Apart from the high-mass stars in the cluster centers, Fig. 2 illustrates that in the models the majority of massive sources outside the cluster centers are also located along the tidal arms while the tidal arms are mostly constructed from low and intermediate mass stars.

A contour density map, which is built from the same realization of the best-matching model, is shown in Fig. 3 along with the population of observed isolated massive stars. These sources fill two stripes along the Galactic plane with a distinct gap in the separation space between the two clusters along the direction of the galactic poles. The observed configuration is closely reproduced in the model by the location of the tidal arms of the two clusters. In Fig. 3, the Nuclear cluster is located at the origin, $(0,0)$. As the minor contribution of the Nuclear cluster was justified in Sect. 3.2, our models do not include the Nuclear cluster.

A group of observed isolated massive stars is located outside the $\operatorname{Pa} \alpha$ survey area around $x \sim-80 \mathrm{pc}$ in Fig. 3. These stars along with more X-ray emitting sources, are thought to be associated with the Sagittarius B molecular cloud complex (Mauerhan et al. 2010b). Figure 3 illustrates that the tidal arms of the Quintuplet cluster might extend out to the Sagittarius B region and therefore the observed evolved massive stars in this region might originate from the tidally drifted structures of the Quintuplet cluster.

The predicted number of massive stars based on the full set of ten different random realizations of each model is presented in Table 3 . The models with an initial mass of $20 M_{\odot}$ for WR progenitors overpredict the expected number of WR stars, which should have been detected in the GC region, while $M>60 M_{\odot}$ provides too few sources to represent the observed population. As Table 3 shows, $M>40 M_{\odot}$ is likely the most realistic initial mass of the progenitor sources that evolve to the observed 


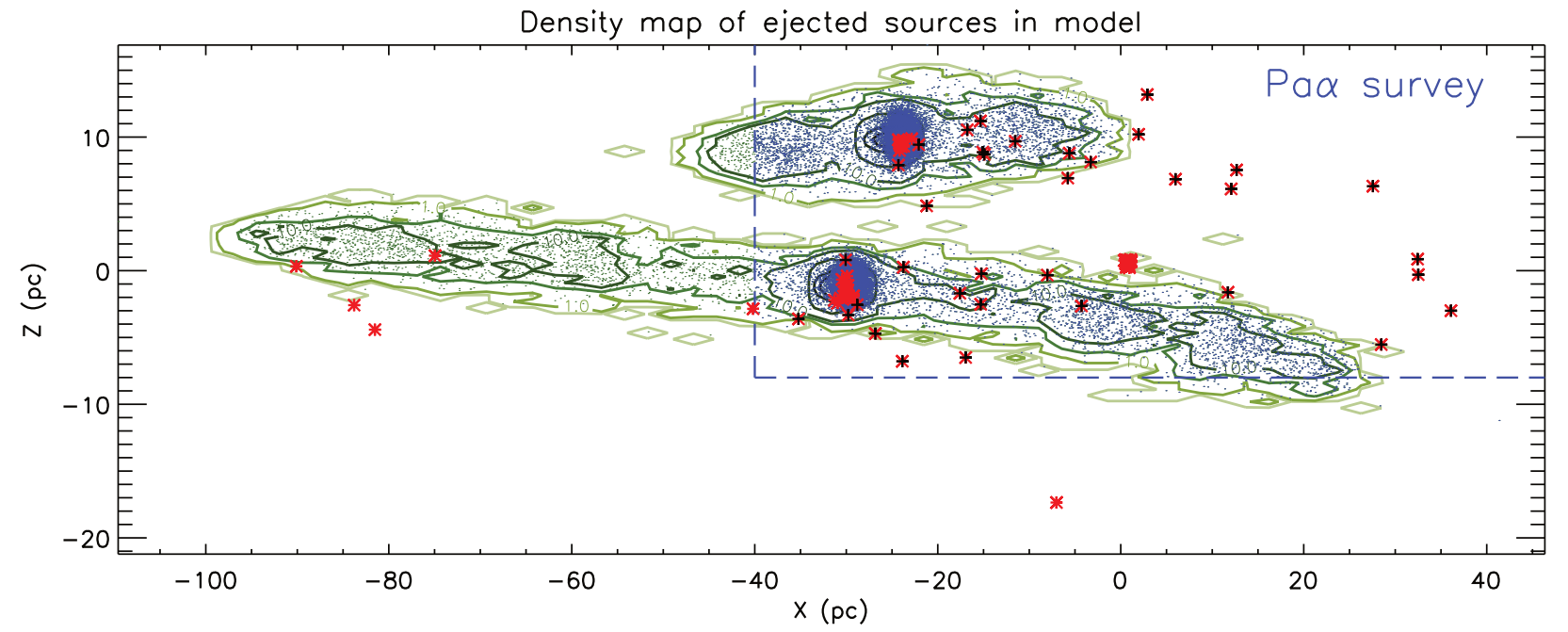

Fig. 3. Contour density map for one of the realizations of the best-matching model with an age of 2.5 and 5 Myr for the Arches and Quintuplet clusters, respectively, and an initial mass of $40 M_{\odot}$ for massive stars is presented with different shades of green lines. Darker colors indicate higher densities. The isolated massive stars observed by Mauerhan et al. (2010a) are marked with red asterisks. The dashed lines approximately represent the border of the Pa $\alpha$ survey. This line separates the region where we perform our comparison between the model and the data. We include some of the observed sources outside the Pa $\alpha$ survey area for illustration. Only the observed sources, as shown by red asterisks, which are marked with black crosses are used to compare observations to the models. Blue (green) dots represent the simulated cluster members, which are included (excluded) for the comparison analysis. The $x$-axis is along the Galactic plane, and the $z$-axis is toward the Galactic north pole.

isolated high-mass population today. These models predict 2230 isolated high-mass stars which cover a significant fraction of the 35 observed massive stars in the $\mathrm{Pa} \alpha$ survey region outside the three clusters (outside their approximate tidal radius) in the GC region.

\subsection{Comparing the spatial distributions}

Table 3 allows us to perform a comparison between the number of massive stars outside the clusters in the models and the observations, which are independent of their spatial distribution. To compare the spatial distribution of drifted sources in the models with the data, we use two different methods. In Sect. 4.2.1, we present the predicted density map of massive stars based on our best-matching model. In Sect. 4.2.2, the feature vector of simulated sources is constructed on the full set of ten different random realizations of the best-matching model (see Sect. 3.3.2) and is compared with the data.

\subsubsection{The density map}

We create a density map of the observed massive stars using Voronoi diagrams. Voronoi tessellations define a neighborhood for each star, the Voronoi cell, on the plane of the sky. Each cell contains one observed star and the set of points on the plane of the sky which are closest to the generating star of the cell. Employing Voronoi diagrams allows us to see the hidden spatial structure in the spread of the points. For example, a preferred orientation in the distribution of points reflects as oriented polygons (Aurenhammer \& Klein 2000).

Figure 5 illustrates the resulting density map. The density map shows two stripes of polygons formed beside the clusters and along the $x$-axis, which confirm the preferred orientation of the observed massive stars along the clusters' tidal tails. The colors of the cells in Fig. 5 are assigned by the calculated probability of observing one or more stars in each particular cell, according to our best-matching model. To calculate the probabilities, we count the average number of predicted modeled massive stars, $M>40 M_{\odot}$, in each Voronoi cell, for all the available random realizations of the model. The corresponding probabilities are accordingly calculated by assuming a Poisson distribution, $P(n \geq 1)=\sum_{i=1}^{\infty} P(n)$. The Poisson probability, $P(n)=\lambda^{n} \mathrm{e}^{-\lambda} / n$ !, yields the probability of observing $n$ stars where the model predicts the mean value, $\lambda$, for the number of stars in each Voronoi cell.

Figure 5 illustrates that the area with a high probability of observing one or more stars is located at a distance of $\sim 20 \mathrm{pc}$ from the cluster centers. The regions, which contain the cluster central members are discarded from the probability calculations (see white regions in Fig. 5). The model predicts relatively high probabilities, $P(n \geq 1)$, along the tidal arms and specifically at the end of the arms. The end of the tidal arms are located at $(x \sim 0, y \sim 10)$ pc for the Arches cluster and $(x \sim 35, y \sim-7)$ pc for the Quintuplet cluster. Figure 5 also shows that $62 \%$ of the observed isolated massive stars, at least one of the ten random realizations of our model predicts a star that can explain the observed star. This number increases to $72 \%$ when we only consider the Voronoi cells within the central $20 \mathrm{pc}$ from the center of the Arches cluster.

The areas of low probabilities are located in the upper right of Fig. $5,10 \lesssim x \lesssim 40,0 \lesssim y \lesssim 10$ pc, and also in the region, which lies between the two clusters, $3<y<7$ pc. These are the sources which, according to our models, are less probable to originate in the tidal arms of the Arches and Quintuplet clusters. In Sect. 4.3, we discuss the possible alternative and parallel scenarios that may explain the origin of these sources. Computed probabilities to observe exactly one star, $P(n=1)$, and to observe one or more stars, $P(n \geqslant 1)$ are presented in Table 2 . It is important to notice that the maximum value of the Poisson probability for observing one star, $P(1)=\lambda \mathrm{e}^{-\lambda}$, is $\sim 37 \%$.

\subsubsection{Comparing feature vectors}

Figure 4 illustrates histograms of distances of all the massive sources with reference to the center of the Arches cluster. It shows that our models produce drifted massive sources at a 
Table 2. Positions and spectral types of the observed isolated massive sources in the GC region within the area covered by the Pa $\alpha$ survey.

\begin{tabular}{|c|c|c|c|c|c|c|}
\hline ID & $\begin{array}{c}\text { RA } \\
\text { (deg, J2000) }\end{array}$ & $\begin{array}{c}\text { Dec } \\
(\mathrm{deg}, \mathrm{J} 2000)\end{array}$ & $\begin{array}{c}\text { Spectral } \\
\text { type }\end{array}$ & References & $P(1)$ & $P(\geq 1)$ \\
\hline 1 & 266.2797 & -29.199789 & WC & 1 & 0.000 & 0.000 \\
\hline 2 & 266.34123 & -29.199841 & WC & 1 & 0.335 & 0.777 \\
\hline 3 & 266.29086 & -29.236897 & WC & 1 & 0.268 & 0.330 \\
\hline 4 & 266.35067 & -29.01608 & OB & 1 & 0.222 & 0.259 \\
\hline 5 & 266.38129 & -28.954669 & $\mathrm{OB}$ & 1 & 0.222 & 0.259 \\
\hline 6 & 266.38549 & -29.082757 & WC & 1 & 0.311 & 0.817 \\
\hline 7 & 266.42203 & -28.863311 & OIf & 1 & 0.222 & 0.259 \\
\hline 8 & 266.42639 & -28.879828 & OB & 1 & 0.090 & 0.095 \\
\hline 9 & 266.47251 & -28.827035 & WN & 1 & 0.193 & 0.926 \\
\hline 10 & 266.50699 & -28.920983 & OI & 1 & 0.164 & 0.181 \\
\hline 11 & 266.51091 & -28.903941 & WC & 1 & 0.359 & 0.551 \\
\hline 12 & 266.54181 & -28.925694 & WN & 1 & 0.090 & 0.095 \\
\hline 13 & 266.57324 & -28.884391 & WN & 1 & 0.090 & 0.095 \\
\hline 14 & 266.59932 & -28.803129 & WN & 1 & 0.002 & 1.000 \\
\hline 15 & 266.54652 & -28.818221 & WN & 1 & 0.031 & 0.994 \\
\hline 16 & 266.4607 & -28.957282 & WC & 1 & 0.222 & 0.259 \\
\hline 17 & 266.52344 & -28.858866 & LBV & 1 & 0.368 & 0.632 \\
\hline 18 & 266.26195 & -29.14986 & $\mathrm{OB}$ & 2 & 0.000 & 0.000 \\
\hline 19 & 266.28733 & -29.20495 & WN & 2 & 0.000 & 0.000 \\
\hline 20 & 266.31744 & -29.0543 & WN & 2,4 & 0.000 & 0.000 \\
\hline 21 & 266.31969 & -28.97364 & WN & 2 & 0.000 & 0.000 \\
\hline 22 & 266.32974 & -29.05609 & WC & 2 & 0.090 & 0.095 \\
\hline 23 & 266.34453 & -28.97895 & WN & 2 & 0.090 & 0.095 \\
\hline 24 & 266.38652 & -28.93797 & OI & 2 & 0.329 & 0.451 \\
\hline 25 & 266.40056 & -28.94405 & WN & 2 & 0.000 & 0.000 \\
\hline 26 & 266.40538 & -28.89827 & OB & 2 & 0.354 & 0.727 \\
\hline 27 & 266.48067 & -28.85738 & WN & 2,3 & 0.090 & 0.095 \\
\hline 28 & 266.57119 & -28.85871 & Of & 2,3 & 0.222 & 0.259 \\
\hline 29 & 266.5743 & -28.83541 & WC & 2 & 0.366 & 0.667 \\
\hline 30 & 266.46089 & -28.98879 & WN & $2,4,5$ & 0.359 & 0.551 \\
\hline 31 & 266.45185 & -28.834709 & WN & 5 & 0.231 & 0.900 \\
\hline 32 & 266.42696 & -28.881472 & WC & 6 & 0.090 & 0.095 \\
\hline 33 & 266.49075 & -28.912806 & WC & 6 & 0.268 & 0.330 \\
\hline 34 & 266.56456 & -28.838492 & WC & 7 & 0.361 & 0.699 \\
\hline 35 & 266.41381 & -28.88923 & $\mathrm{OB}$ & 4,5 & 0.222 & 0.259 \\
\hline
\end{tabular}

Notes. From left to right, the columns are as follows: Sequential ID for stars, RA, Dec, spectral type, and the reference study. The last two columns of the table present the Poisson distribution, $P(n)=\lambda^{n} \mathrm{e}^{-\lambda} / n$ !, of an observing one star, $P(1)$, and one or more stars, $P(n \geq 1)=\sum_{i=1}^{\infty} P(n)$, in the assigned Voronoi cell of each particular star on the density map of the observed isolated massive stars illustrated in Fig. 5. It is important to notice that the maximum value of the Poisson probability of observing one star, $P(1)=\lambda \mathrm{e}^{-\lambda}$, is $\sim 37 \%$.

References. (1) Mauerhan et al. (2010a); (2) Mauerhan et al. (2010b); (3) Mauerhan et al. (2007); (4) Muno et al. (2006); (5) Cotera et al. (1999); (6) Homeier et al. (2003); (7) Figer et al. (1999b).

Table 3. The predicted number of massive stars of each model.

\begin{tabular}{ccccccc}
\hline \hline \multicolumn{7}{c}{$N_{\text {massive }}$ outside the clusters } \\
\hline & WR-L $=20 M_{\odot}$ & StdDev & WR-L $=40 M_{\odot}$ & StdDev & WR-L $=60 M_{\odot}$ & StdDev \\
\hline$Q_{\text {age }}=4 \mathrm{Myr}$ & 62 & $\sigma_{4,20}=8$ & $\mathbf{2 2}$ & $\sigma_{4,40}=5$ & 11 & $\sigma_{4,60}=4$ \\
$Q_{\text {age }}=4.5 \mathrm{Myr}$ & 80 & $\sigma_{4.5,20}=12$ & $\mathbf{3 0}$ & $\sigma_{4.5,40}=4$ & 13 & $\sigma_{4.5,60}=2$ \\
$Q_{\text {age }}=5.0 \mathrm{Myr}$ & 68 & $\sigma_{5,20}=8$ & $\mathbf{2 6}$ & $\sigma_{5,40}=3$ & 10 & $\sigma_{5,60}=2$ \\
\hline
\end{tabular}

Notes. In the Pa survey region, 35 massive stars are observed outside the three clusters (outside their approximate tidal radius) in the GC region. Among these massive stars are 24 Wolf-Rayet stars.

distance up to $70 \mathrm{pc}$ from the Arches center. This result implies, that the possibility of being a run-away/drifted star especially from the Quintuplet cluster cannot be excluded for an observed isolated massive star at a distance up to $70 \mathrm{pc}$ from the Arches center. The provided list by Mauerhan et al. (2010a) includes $11 \mathrm{WC}$ and $13 \mathrm{WN}$ stars with $1 \mathrm{LBV}$ and $10 \mathrm{OB}$ supergiants outside the $1.6 \mathrm{pc}$ radius of the three clusters in the $\mathrm{Pa} \alpha$ survey region. The comparison of their full list in the $\mathrm{Pa} \alpha$ survey area to the model is shown in Fig. 4. Observed sources, which lie in the central $1.6 \mathrm{pc}$ of the three clusters, i. e. Arches, Quintuplet, and Nuclear cluster, are excluded from these histograms. The bestmatching model reproduces $80 \%$ of the known isolated massive stars out to a distance of $21 \mathrm{pc}$ and $67 \%$ of the observed population out to $80 \mathrm{pc}$ from the center of the Arches cluster. However, since the population of $\mathrm{OB}$ supergiants is not complete in this catalog, we excluded all the OB stars from the original observed list and repeated the comparison. This comparison shows that our model produces $20 \%$ more massive sources 
M. Habibi et al.: Isolated massive population in the Galactic center
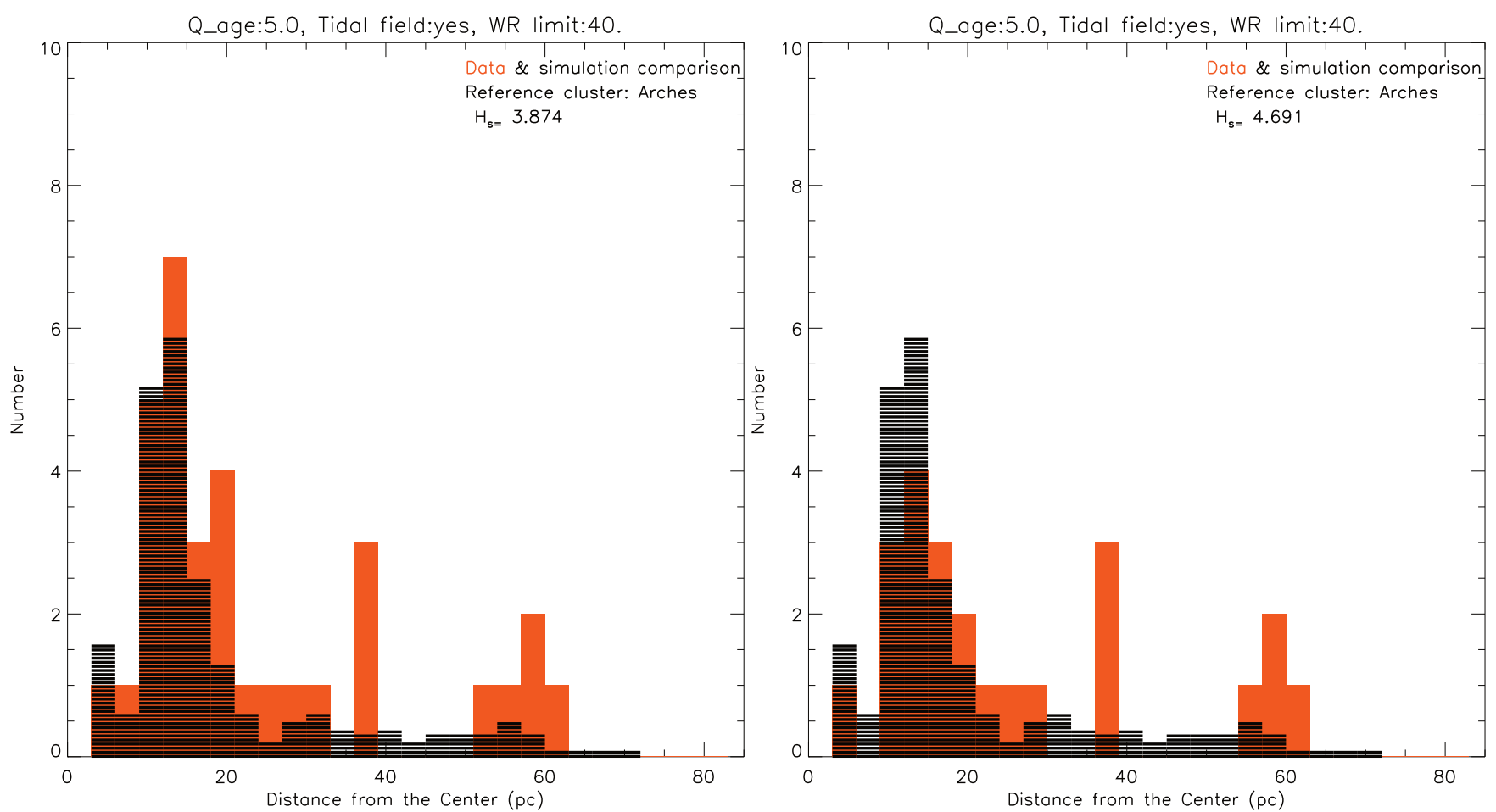

Fig. 4. Line-filled histograms depict the average distribution of the massive sources, $M>40 M_{\odot}$, in the ten random realizations of the bestmatching model. Stars within the central $1.6 \mathrm{pc}$ of the simulated Arches and Quintuplet clusters are excluded. Red filled histograms illustrate the distribution of the observed massive sources. Distances are calculated with reference to the center of the Arches cluster. Left: the comparison is based on the full list of WR stars and OB supergiants presented by Mauerhan et al. (2010a). Right: OB super giants are excluded from the observed catalog and the comparison is based on the population of WRs in the region.
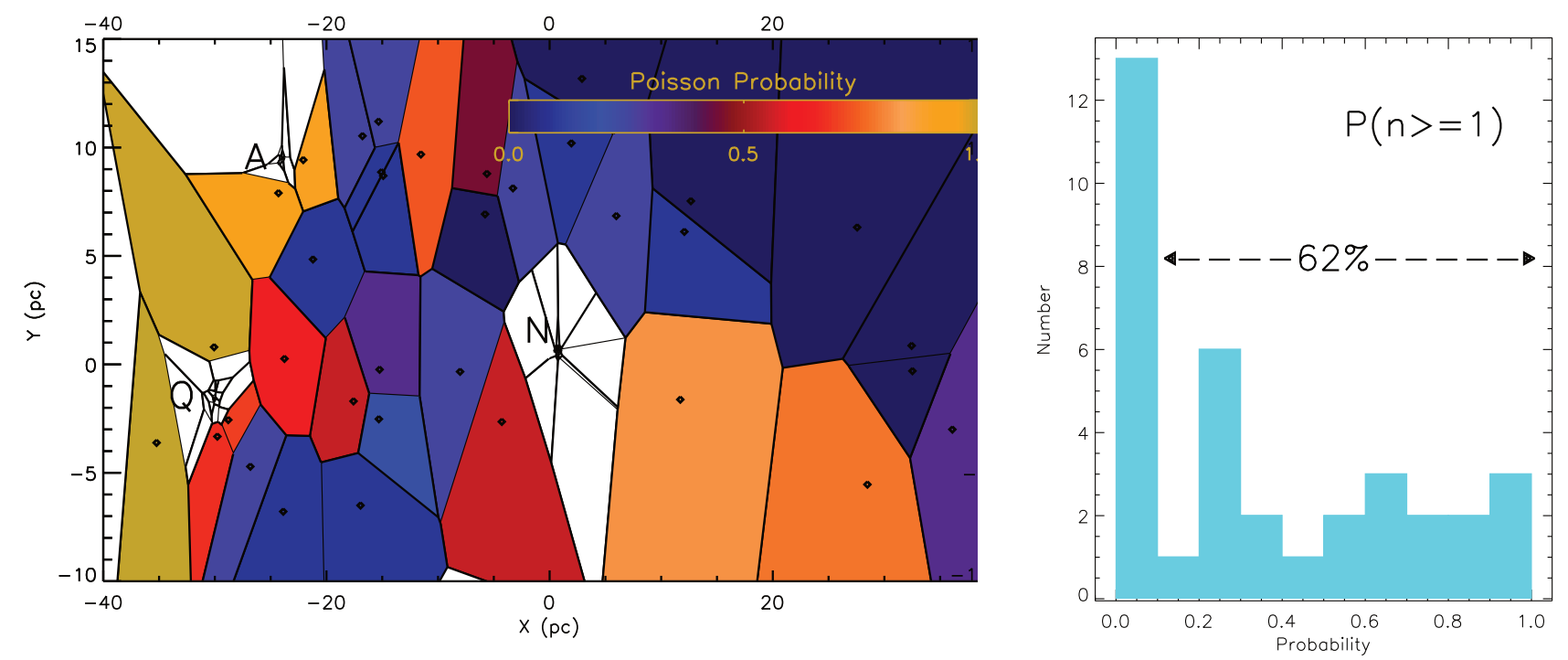

Fig. 5. Left: density map of the observed massive stars (Mauerhan et al. 2010a) is derived using Voronoi diagrams. The Voronoi cells are colored according to their calculated Poisson probability of observing one or more stars in each cell, $P(n \geq 1)$, based on our best model. The color-bar assigns colors to the calculated probabilities. White regions contain the cluster central members and are discarded from the probability calculations. Right: distribution of calculated Poisson probabilities, $P(n \geq 1)$, is shown. Our model reproduces at least one star in ten random realizations, $P(n \geq 1)>10 \%$, for $62 \%$ of the observed isolated massive stars. Please note that the Voronoi cells appear skewed due to different scales for the $x$ and $y$ axis.

as compared to the observed population of $\mathrm{WN}, \mathrm{WC}$, and $\mathrm{LBV}$ stars in the GC region out to a distance of $21 \mathrm{pc}$ from the center of the Arches cluster (see Fig. 4, right). This over-prediction may occur because we can only choose an initial stellar mass. We have no means to distinguish the spectral types of the stars in our models. Hence, stars, which are in the supergiant phase or even main sequence phase, are always partially included. It can also be due to a missing population of WC stars. The Pa $\alpha$ line strength of both WN and WC stars span a similar range of $1.2<F 187 / F 190 N<2.9$ (Mauerhan et al. 2010a). The bright $K_{\mathrm{s}}$ counterparts of WN stars guarantees a near completeness of the WN sample in the GC. However, due to the faint end of the $K_{\mathrm{s}}$ brightness distribution of observed WC stars, their sample is probably not complete (see Mauerhan et al. 2010a for details). 


\subsection{Alternative scenarios}

In both histograms of Fig. 4, the major peak in the observed distribution of isolated massive stars at $\sim 15 \mathrm{pc}$ is well reproduced by the best-matching model. Nevertheless, the two minor peaks at distances of $\sim 40 \mathrm{pc}$ and $\sim 60 \mathrm{pc}$ from the Arches center are absent in the model. Out of the 35 observed massive sources, 27 stars are located close to the modeled tidal arms of either of the two clusters. The remaining eight sources are observed outside the modeled arms. These sources, which are not reproduced with our models can have different origins: many high mass stars are expected to be in binaries with high-mass companions $(\sim 70 \%$ of all $\mathrm{O}$ stars of which $\sim 40 \%$ are expected to have evolved with strong interactions; Sana et al. 2012). The observed sources outside the tidal structures can originate in tight binary systems in which the primary has already exploded as a supernova (Blaauw 1961; Stone 1991). Therefore, the secondary has received a kick and is ejected at a random direction and at a high relative velocity compared to the cluster orbital velocity. These ejecta are expected to have a high velocity compared to the velocity of the cluster center and can possibly explain the detected population in between the modeled tidal structures and the single detected massive star at $z=-18$ pc (see Fig. 3). This process is especially expected from some of the most massive stars in the Quintuplet cluster at the suggested age of 4 to $5 \mathrm{Myr}$. Another possibility for their origin is that they are formed independently of the current observed clusters.

A possible Orion nebula (ONC)-type host star cluster, which owns $\sim 1$ massive source of $\sim 35 M_{\odot}$ cannot be detected in the GC region with any of the current wide-field surveys. The density of such a star cluster is too low, and intermediate-mass sources $\left(M<10 M_{\odot}\right)$ are too faint to stand out among the dense, crowding-limited GC field. These clusters are expected to dissolve even faster than the young sturburst clusters. The survival lifetime of less than $10 \mathrm{Myr}$ suggests that they could appear too dispersed to be detected as a compact entity, such as in the Arches cluster (for discussion on dissolution time and detectability of the clusters in the GC, see Portegies Zwart et al. 2001a). The other scenario is that these sources are formed in isolation from the dense molecular clouds observed in the central molecular zone (Longmore et al. 2012). The last two scenarios are currently hard to distinguish.

The discrepancies between the model and the data also can originate in limitations of our models. In the absence of precise $3 \mathrm{D}$ velocity measurements for the Quintuplet cluster ${ }^{4}$, our models assume that the Quintuplet cluster is an older representation of the Arches cluster. Although this assumption is based on the observed density and age of the Quintuplet cluster, a more accurate measurement of the 3D velocity of the Quintuplet cluster, will eventually help in choosing the best matching orbit for the Quintuplet. Employing different orbits can shift the position of the tidal tails on the plane of the sky. We expect that our experiment that assumes three different ages for the Quintuplet cluster partly covers the effect of uncertain orbit of the Quintuplet cluster. The other limitation of the models is the lack of primordial binaries. Although wide binaries form automatically in the models in the early stages of cluster evolution, the population of tight initial binaries is missing in these simulations. These

\footnotetext{
4 The preliminary analysis of the proper motion data of the Quintuplet cluster yields $172 \pm 50 \mathrm{~km} \mathrm{~s}^{-1}$ (Stolte et al. 2011), which is surprisingly similar to the measured value of $172 \pm 15 \mathrm{~km} \mathrm{~s}^{-1}$ for the Arches cluster (Clarkson et al. 2012). Given the close projected distance between the Arches and Quintuplet clusters in the GC region, we hence use the Arches orbit to simulate the Quintuplet cluster.
}

binaries play an important role in dynamical three or four-body encounters, which produce ejected stars (e.g., Poveda et al. 1967; Gies \& Bolton 1986). Adding primordial binaries to these models produce more massive sources outside the tidal radius of the clusters and strengthen the similarity of the models with the data. These sources are less likely to be spatially confined to the tidal arms of the cluster, yet the effect of the GC tidal field on these high-velocity ejected sources needs a thorough investigation in future studies.

In summary, the dynamical model of the Arches and Quintuplet clusters, which assumes an initial mass of $40 M_{\odot}$ for the WR star progenitors and a Quintuplet age of $5 \mathrm{Myr}$, can explain up to $80 \%$ of the observed isolated population of massive stars.

\subsection{The velocity distribution of drifted cluster members}

Tidal arms form as a result of differential velocities produced by the many body dynamical interactions between stars in the cluster. Since the cluster moves along its orbit, this velocity difference causes stars to either fall behind or take over the cluster center. Figure 6 illustrates the velocity variation in the Arches (top panel) and the Quintuplet clusters (bottom panel). The velocity variation of the stars in the tidal arms reaches $50 \mathrm{~km} \mathrm{~s}^{-1}$ for the Arches cluster and $140 \mathrm{~km} \mathrm{~s}^{-1}$ for the Quintuplet cluster. The distribution of the massive stars, $M>40 M_{\odot}$, in Fig. 7 shows that there are sources with masses of up to $100 M_{\odot}$ in the furthest extent of the tidal tails, despite that most of the massive stars sink to the cluster core. For example in the illustrated model in Fig. 7, a $100 M_{\odot}$ star is found at the distance of $\sim 15 \mathrm{pc}$ from the Arches and $\sim 60 \mathrm{pc}$ from the Quintuplet center. Most of the massive sources, which are outside the central tidal region have velocities similar to the tidally diffused population around them. The few massive sources that lie outside the tidal structure have velocities similar to the extreme velocities of stars in the tidal arms. To study the mechanism allowing the sources to drift out of the cluster in more detail, we analyze the trajectories of the sources in different mass ranges and compare them to the position of the cluster on its orbit around the GC. Stolte et al. (2008a) showed that the Arches cluster evolves along an open eccentric rosetta-like orbit (see Figs. 9a and A.1a). Figure 8a demonstrates the variation of Galactocentric distance of the cluster over time. As the cluster moves along its orbit, it encounters a spatially varying tidal field caused by the GC potential and its asymmetric orbit. The corresponding pericenters and the apocenters ${ }^{5}$ on each period are indicated in Figs. 8 and 9a. The projected orbit of the cluster in the $x y$-plane, which is close to the orbital plane of the modeled cluster, is indicated in Fig. 9a; the projected snapshots of the cluster at apsides are illustrated in Fig. 9b, which allow us to follow the spatial spread and the velocity variation of the cluster along its orbit without being biased by the projection of the cluster on the plane of the sky, the $x z$-plane.

Following the pericenters and the apocenters on the orbit, we can trace the trajectory of the cluster members with respect to the cluster center (see Fig. 8). Both groups of massive stars, $M>40 M_{\odot}$, and stars with intermediate masses, $10 M_{\odot}<M<20 M_{\odot}$, follow a similar pattern (Figs. 8b and 8c). Our finding that velocities of the simulated massive sources are in the range of the velocity variation along the tidal arms

\footnotetext{
5 As the cluster is not on an elliptical eccentric orbit, we use the word pericenter (apocenter) only to refer to the point of closest (furthest) approach to the center of the potential on each orbit. The position of these points are different for each period.
} 
M. Habibi et al.: Isolated massive population in the Galactic center
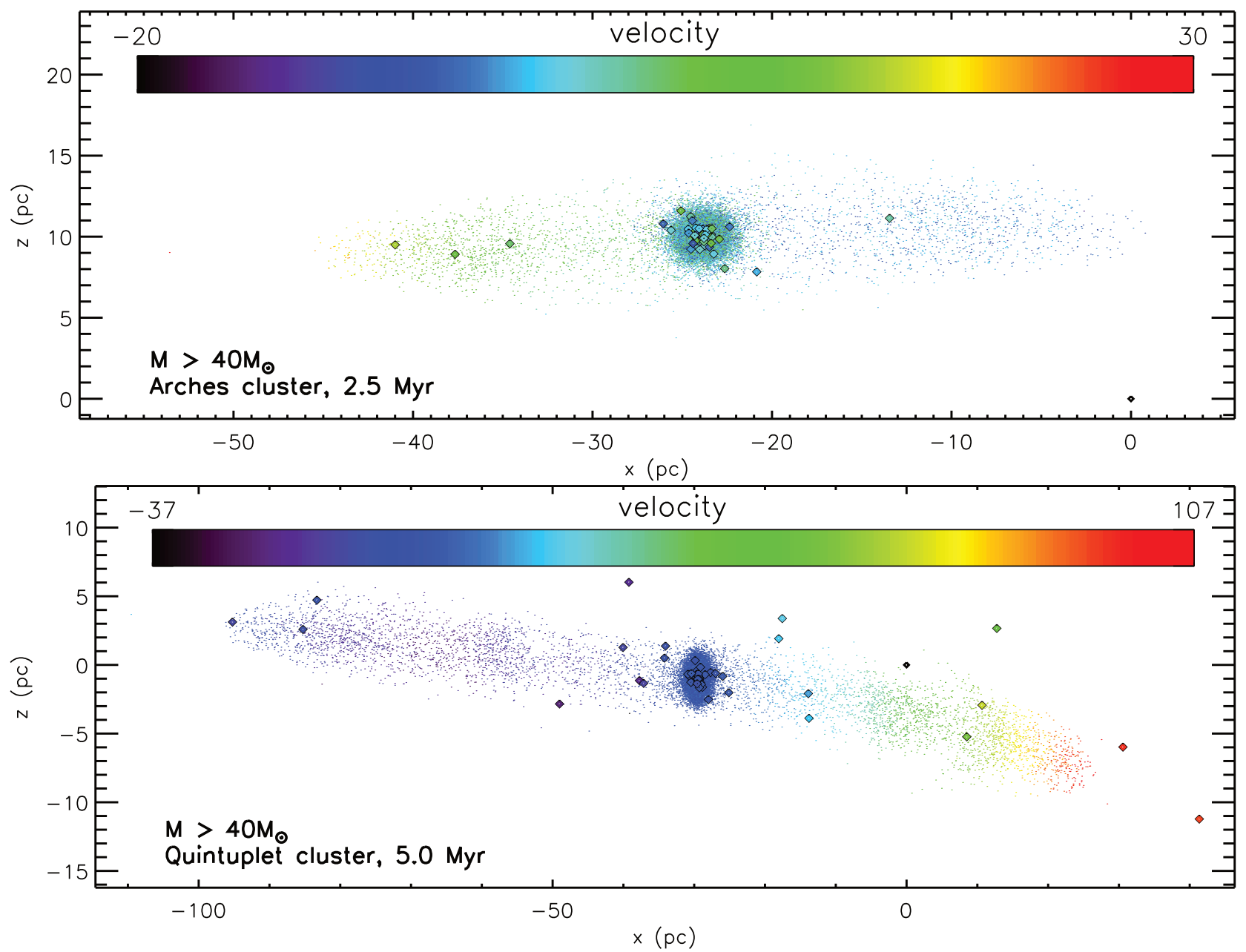

Fig. 6. Velocity variation within the tidal arms for one realization of the simulated cluster at an age of $2.5 \mathrm{Myr}$ (up) and $5 \mathrm{Myr}$ (down). Massive stars, $M>40 M \odot$, are illustrated with larger symbols. Cluster members are color coded according to their velocities along the Galactic plane, $V_{x}$.
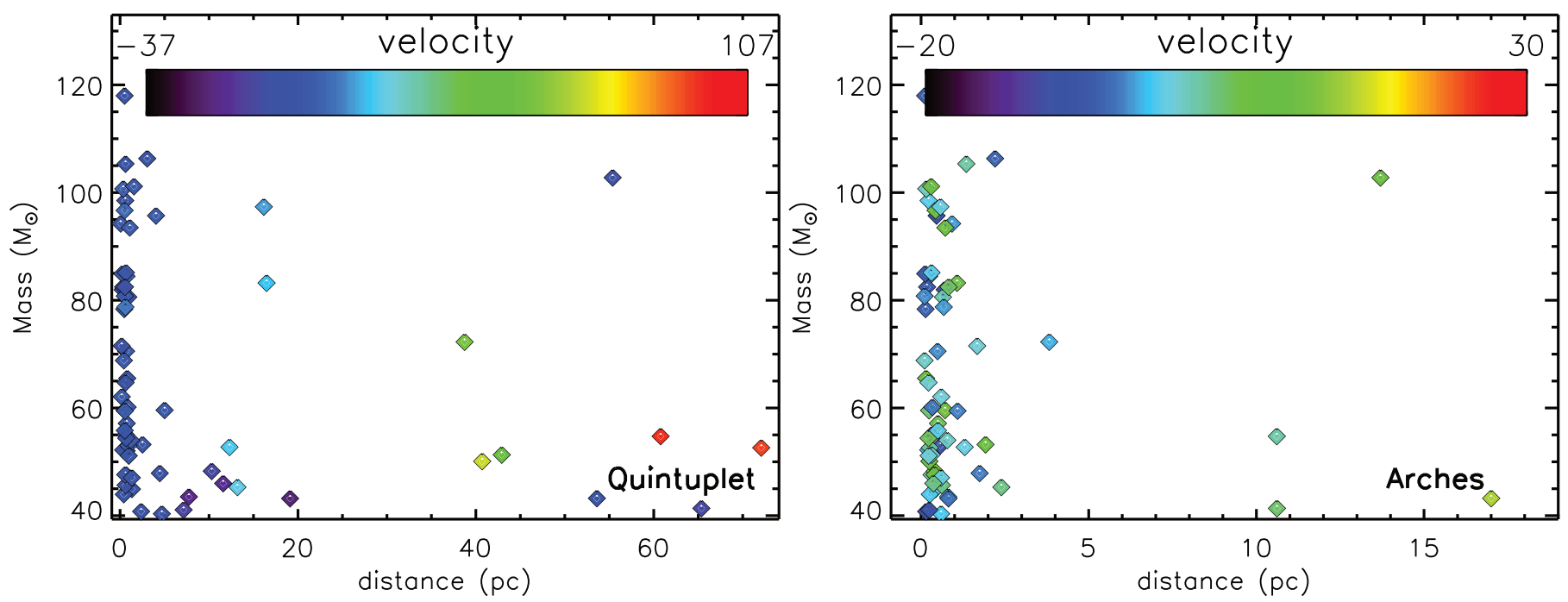

Fig. 7. Initial masses are plotted over the distance from the cluster center for the massive stars, $M>40 M_{\odot}$. Stars are color coded according to their velocities along the Galactic plane, $V_{x}$. Plotted populations are for one of the realizations of the model.

shows, that high-mass and intermediate-mass stars outside the tidal radius of the cluster gain energy through similar physical processes. Some of the stars gain energy in the center of the cluster probably through two-body relaxation. Afterward, as these sources recede from the cluster center, they undergo several periods where they slightly fall toward the cluster and are followed by pulling away from the cluster center. During the time that the cluster moves away from the apocenter toward pericenter, its velocity increases, and consequently, individual orbits of stars on the tidal structures diverge spatially. This effect is 


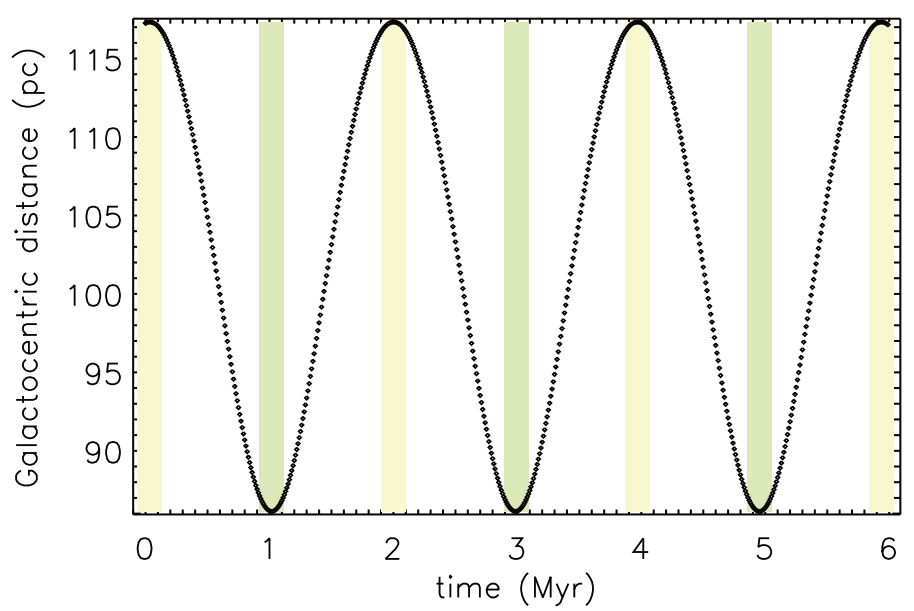

(a)

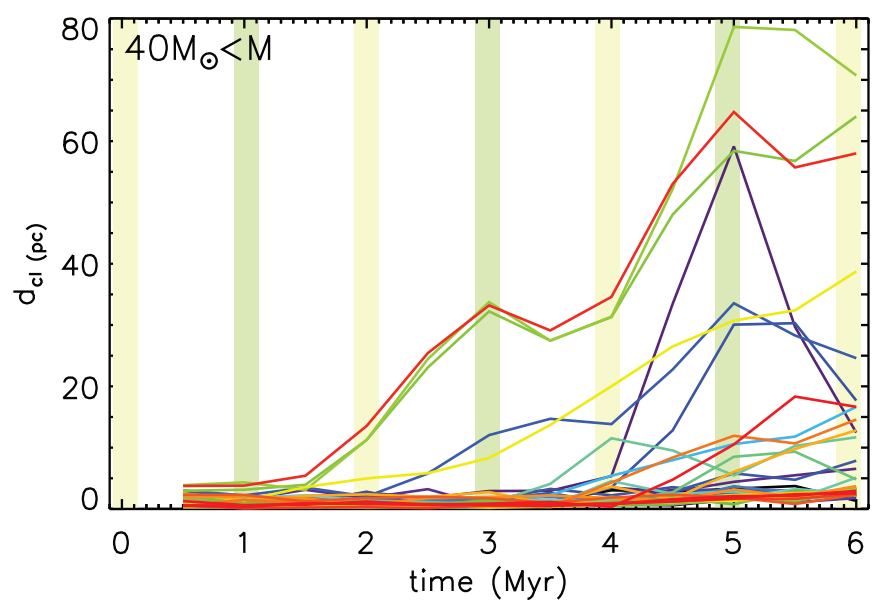

(c)

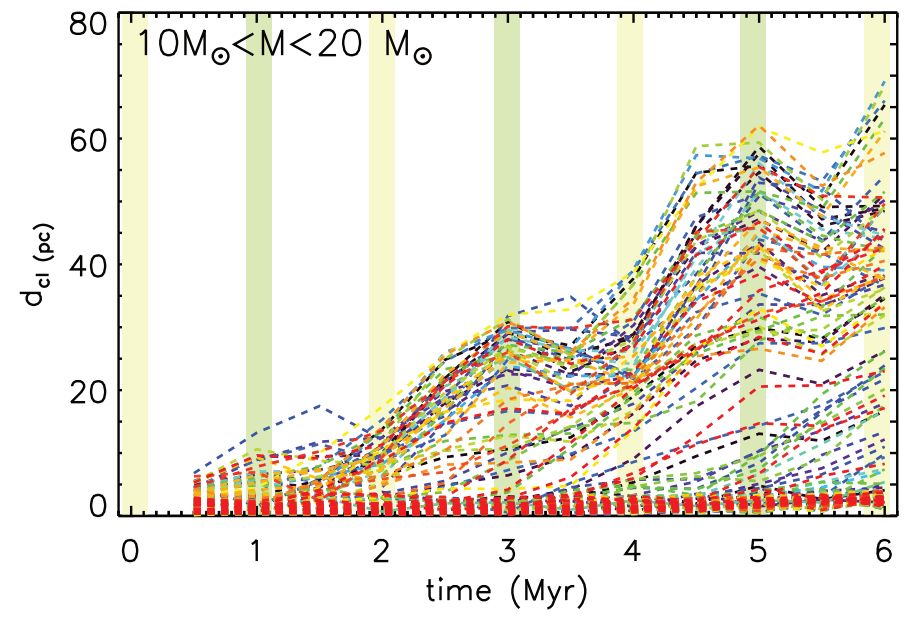

(b)

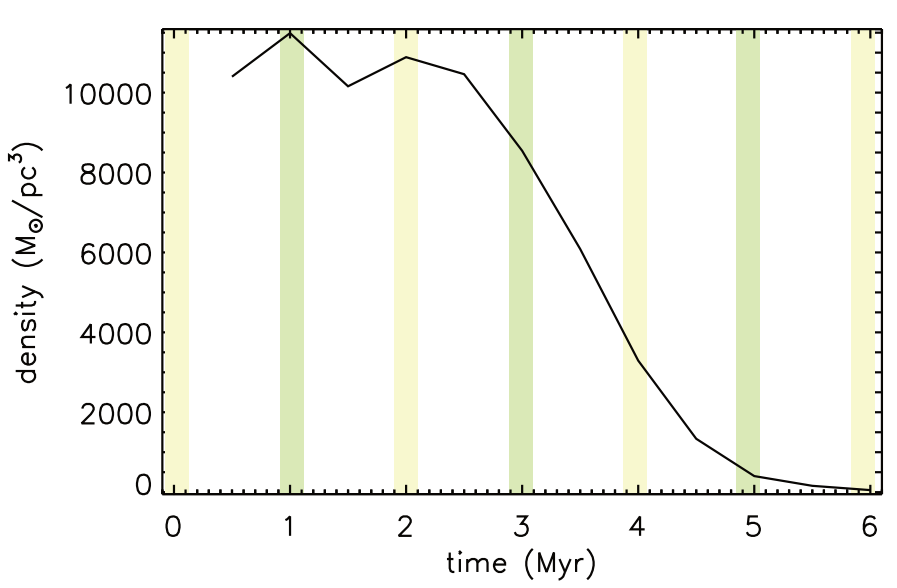

(d)

Fig. 8. a): Projected galactocentric distance of the cluster on the plane of the sky $(x, z)$ is plotted over time. As the cluster moves along its open eccentric orbit, it encounters the variable tidal field caused by the GC. In Figs. a) to d) regions of maximum and minimum Galactocentric distances are marked with yellow and green boxes respectively. b), c): Projected distance of stars on the plane of the sky with reference to the cluster's center, $d_{\mathrm{cl}}$, is plotted over time. Each line represents a trajectory of one star and colors are different to better distinguish lines. Trajectories of the cluster members are illustrated for the two mass ranges of $10 M_{\odot}<M<20 M_{\odot}$ and $M>40 M_{\odot}$ in Figs. b) and c) respectively. d): Surface mass density of the center of the cluster is plotted over time. The density is calculated for a cylinder of $0.5 \mathrm{pc}$ perpendicular to the plane of the sky.

indicated in Fig. 8, as the sources recede from the cluster center after the apocenter passage. The projected snapshots of the cluster on the $x y$-plane that is close to the orbital plane of the cluster illustrate the expansion of the cluster, including its tidal arms, after passing the apocenter and accelerating toward pericenter (see Fig. 9b; compare the snapshots of the cluster on each pericenter to its snapshot at its previous apocenter). Comparably, when the cluster approaches the apocenter and decelerates, stars on the tidal tails contract toward the cluster center (see Fig. 8), and the cluster appears less expanded (see Fig. 9a). A similar behavior is discussed in studies that investigate halo globular clusters and their interaction with the Galactic field through several Gyrs of evolution (e.g., Küpper et al. 2010). We have calculated the surface mass density inside a cylinder of $0.5 \mathrm{pc}$ at the center of the cluster and perpendicular to the plane of the sky, as it can be investigated by observations. The surface mass density of the central region of the cluster declines as the cluster expands on its orbit (see Fig. 8d).

Although snapshots of the cluster along the $x y$-plane and close to the orbital plane of the cluster follow the dynamical evolution of the cluster, the observed cluster is affected by the projection of the cluster on the plane of the sky. Figure A.1 in Appendix A illustrates the projected orbit of the cluster on the plane of the sky with snapshots of the cluster while crossing the apsides.

In summary, the models suggest that high-mass stars drift out of the clusters with the same physical mechanisms as intermediate-mass stars that form the tidal tails. Hence, their velocity is similar to the tail stars, and their location is consistent with the spatial distribution of the "isolated" WR stars close to the simulated location of the tidal tails. Figures $8 \mathrm{~b}$ and $8 \mathrm{c}$ show that the drift pattern of high-mass stars over time is very similar to the drift pattern of intermediate mass stars. They follow the same apo/pericenter variation and are not rapid ejectors in these models. The clusters expand rapidly after $3 \mathrm{Myr}$, so that tidal tails stretch out over 120 pc across the central molecular zone within 5 Myr (see Fig. A.1 in Appendix A and Fig. 9b), which is close to its entire diameter $(\sim 120 \mathrm{pc})$. According to these models, it is therefore not surprising anymore to find isolated highmass stars at large distances from the location of the clusters 
M. Habibi et al.: Isolated massive population in the Galactic center

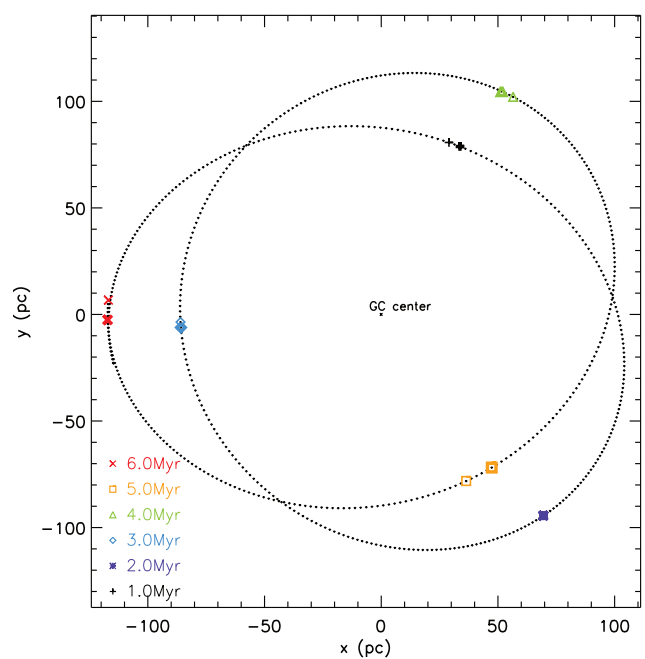

(a)
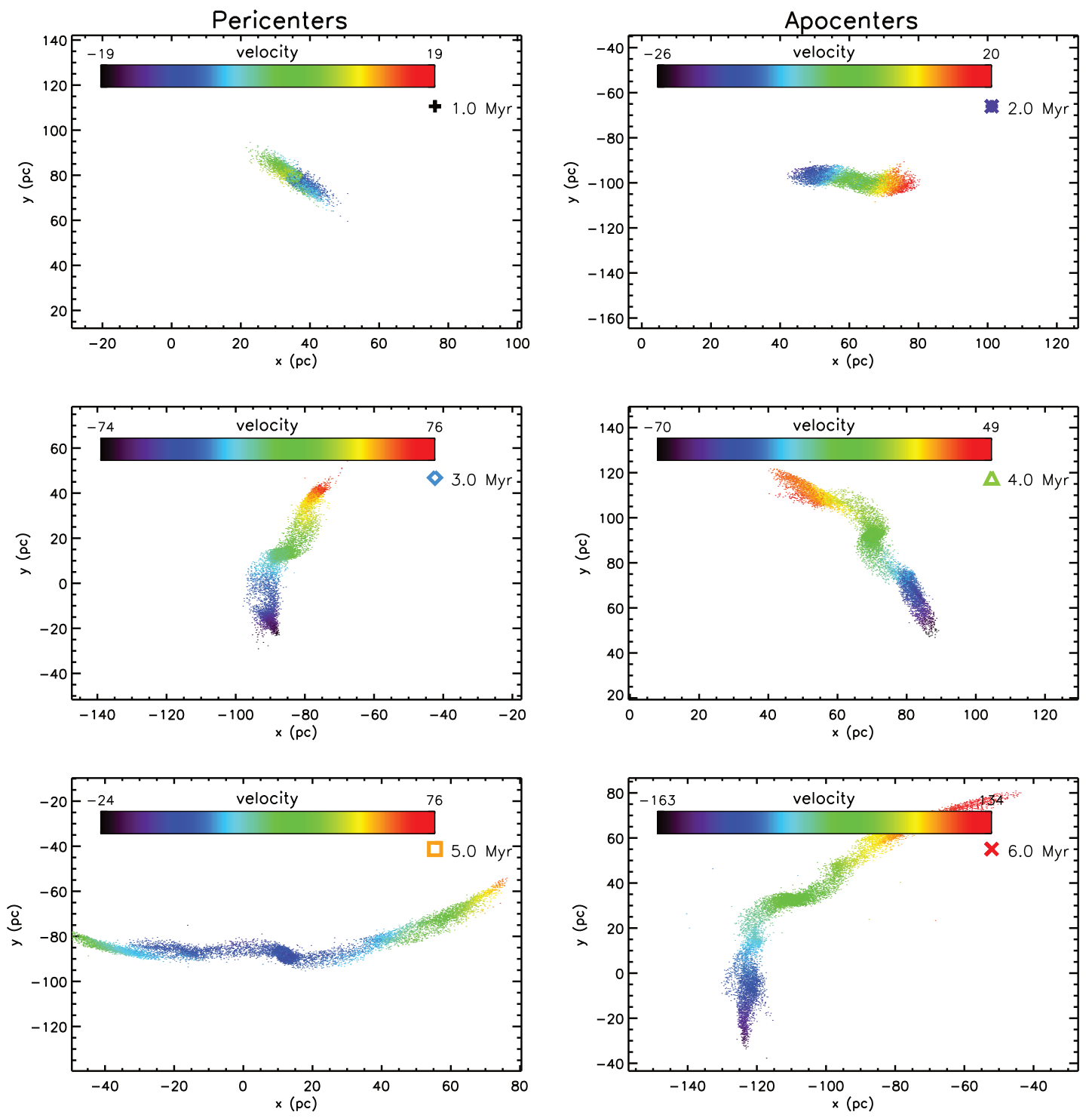

(b)

Fig. 9. a) Projected orbit of the cluster on the $x y$-plane, which is close to the cluster's orbital plane, is illustrated. The symbols on the orbit mark the apsides of the 3D orbit. Each symbol/color corresponds to a particular age. The thick symbols mark the exact position of the apsides, while the thin symbols refer to the closest available snapshot of the simulated cluster by considering the time resolution of the simulations. b) Projected snapshots of the cluster on the $x y$-plane are shown at apocenters and pericenters. Each snapshot corresponds to one marked apside on the projected orbit. The sources are colored based on the present velocity variation in the cluster and its tidal tails. 
today as they drift along the tidal arms; that is, they are expected to occupy the entire area where the tidal structures stretch out.

\section{Conclusions}

In this study, we present $N$-body simulations of the Arches cluster to create combined models of the Arches and Quintuplet clusters. The population of ejected and drifted sources from the two clusters is compared to the HST/NICMOS Paschen- $\alpha(\mathrm{Pa} \alpha)$ survey of the Galactic center, which detects the distribution of young massive stars in the GC region (Mauerhan et al. 2010a). Our study can be summarized as follows:

1. We construct different combined models of massive sources outside the Arches and Quintuplet clusters by assuming different ages for the Quintuplet cluster and distinct values for the initial mass of a WR progenitor (see Table 1). We compare these models to the observed population of massive stars presented by Mauerhan et al. (2010a) by employing a method, which calculates the histogram difference between spatial distributions of stars. Among all the constructed models, the model, which assumes an age of $5 \mathrm{Myr}$ for the Quintuplet and an initial mass of $40 M_{\odot}$ for a WR progenitor, is the most similar to the spatial distribution of the observed isolated high-mass stars.

2. The strong tidal field of the GC potential results in extended tidal arms for both the Arches and Quintuplet clusters at their current age. In the best-matching model, tidal arms of the Arches cluster stretch out to $20 \mathrm{pc}$ in each direction on the plane of the sky and along the Galactic plane, while the tidal arms of the Quintuplet cluster extend out to $65 \mathrm{pc}$. The observations of massive stars in the GC region (Mauerhan et al. 2010a) reveal two strips along the Galactic plane with a prominent gap along the direction of the galactic poles. The projected positions of the tidal structures of the two clusters closely reproduce this observed distribution (see Fig. 3).

3. The observed massive sources outside the three clusters, including the young Nuclear cluster in the Pa $\alpha$ survey area, is compared to the models using two different methods. First, comparing histograms of the spatial distributions of observed and simulated stars shows that the best-matching model reproduces $80 \%$ of the observed population out to $21 \mathrm{pc}$ and $67 \%$ of the observed population out to $80 \mathrm{pc}$ distance with reference to the center of the Arches cluster. Second, we create a density map of observed isolated massive stars using Voronoi diagrams. The constructed density map allows us to probe the probability of observing one or more stars in each Voronoi cell by assuming our best-matching model. For $62 \%$ of the observed isolated massive stars, at least one of the ten random realizations of our model predicts a star that can explain the observed star. This number increases to $72 \%$ when we only consider the Voronoi cells within the central $20 \mathrm{pc}$ from the center of the Arches cluster. The sources that cannot be explained as originating from the Arches and Quintuplet are located at large distances from the tidal tails. On the other hand, the best-matching model predicts $20 \%$ more massive stars outside the clusters, when we perform the comparison between the best-matching model and the list of observed WR stars only, which excludes the less complete sample of OB sources (see Fig. 4).

4. The best-matching model predicts 26 massive stars outside the clusters, as compared to the 35 observed massive stars outside the three clusters in the $\mathrm{Pa} \alpha$ survey region. According to our best-matching models, the majority of the simulated massive sources are located close to the tidal structure of the clusters, while the list of 35 observed massive stars includes 27 sources close to the tidal arms and 8 sources, which are not close to the tidal structure of the Arches and the Quintuplet cluster. Histograms of the spatial distribution of the observed sources display a major characteristic peak at $\sim 15 \mathrm{pc}$ from the center of the Arches cluster. This peak is well reproduced in the best-matching model (see Fig. 4). However, two minor peaks at the distances of $\sim 40 \mathrm{pc}$ and $\sim 60 \mathrm{pc}$ from the Arches center are absent in the model. Possible origins of these sources are supernova kicks or dynamical ejections involving tight initial binary systems. In this case, these additional WR stars could also have emerged from the Arches and Quintuplet clusters. Currently, we cannot also exclude the possibility that these high-mass stars might have formed in smaller clusters or in isolation. Finally, a deviation, especially in the orbit of the Quintuplet cluster, could also give rise to the remaining differences between the observed and the simulated spatial distribution of high-mass stars in the GC.

5. According to our models, the projected tidal arms of the Quintuplet cluster at the age of $5 \mathrm{Myr}$ extends out to $60 \mathrm{pc}$ and reaches to the Sagittarius B2 region. This implies that the evolved massive stars observed in projection toward this region might originate in the tidally drifted sources from the Quintuplet cluster.

6. The tidal structure of both clusters form as a result of velocity variation in the cluster. The velocity variation along the tidal arms of the Arches cluster reaches $50 \mathrm{~km} \mathrm{~s}^{-1}$. This value is as high as $140 \mathrm{~km} \mathrm{~s}^{-1}$ for the Quintuplet cluster, which is detectable in a proper motion diagram of high precision astrometric studies of the Quintuplet cluster.

7. The trajectories of the sources in different mass ranges in our models show that the tidal drifting of the cluster stars by the GC potential is an effective process, which causes the stars to recede out to $70 \mathrm{pc}$ from the center of the Arches cluster. Both massive, $M>40 M_{\odot}$, and intermediate mass stars, $10 M_{\odot}<M<20 M_{\odot}$, follow a similar pattern; they gain energy in the center of the cluster, which causes these sources to exceed the cluster's escape velocity. This suggests that the massive and intermediate-mass stars are evolved from the clusters by the same dynamical processes into tidal tails. The extended radial coverage of the high-mass stars inside the tidal tails implies that up to $80 \%$ of the isolated observed WR population can be explained by cluster stars.

Acknowledgements. M.H. and A.S. acknowledge funding from the German science foundation (DFG) Emmy Noether program under grant STO 496-3/1, and thank the Argelander-Institute for Astronomy at the University of Bonn for being such a generous host. We wish to thank Dr. A. Liermann and Dr. C. Olczak for helpful discussions. We also thank Dr. C. Olczak for careful and substantial comments on the manuscript. We thank Dr. D. Applegate for valuable discussions on statistics. We are grateful to our anonymous referee for comments and suggestions that helped improve the paper.

\section{Appendix A: The projected view on the plane of the sky}

In Fig. A.1, we illustrate the projected orbit of the modeled cluster around the GC on the plane of the sky, the $x z$-plane. As the cluster moves along the 3D orbit, it passes through apsides. These apsides are marked on the orbit with the projected snapshot of the cluster at the corresponding apside point. Comparing Fig. A.1b with Fig. 9b shows the strong effect of the projection. For example, the snapshot of the cluster at the age of $3 \mathrm{Myr}$ 


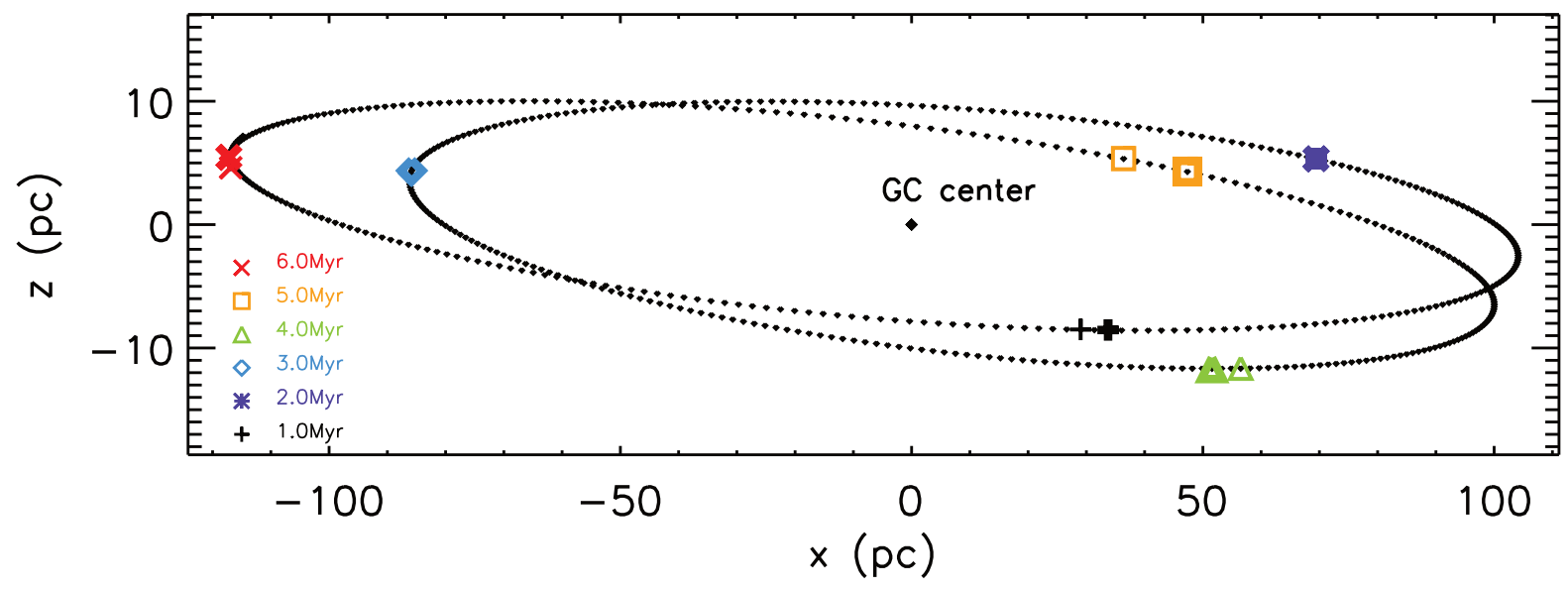

(a)
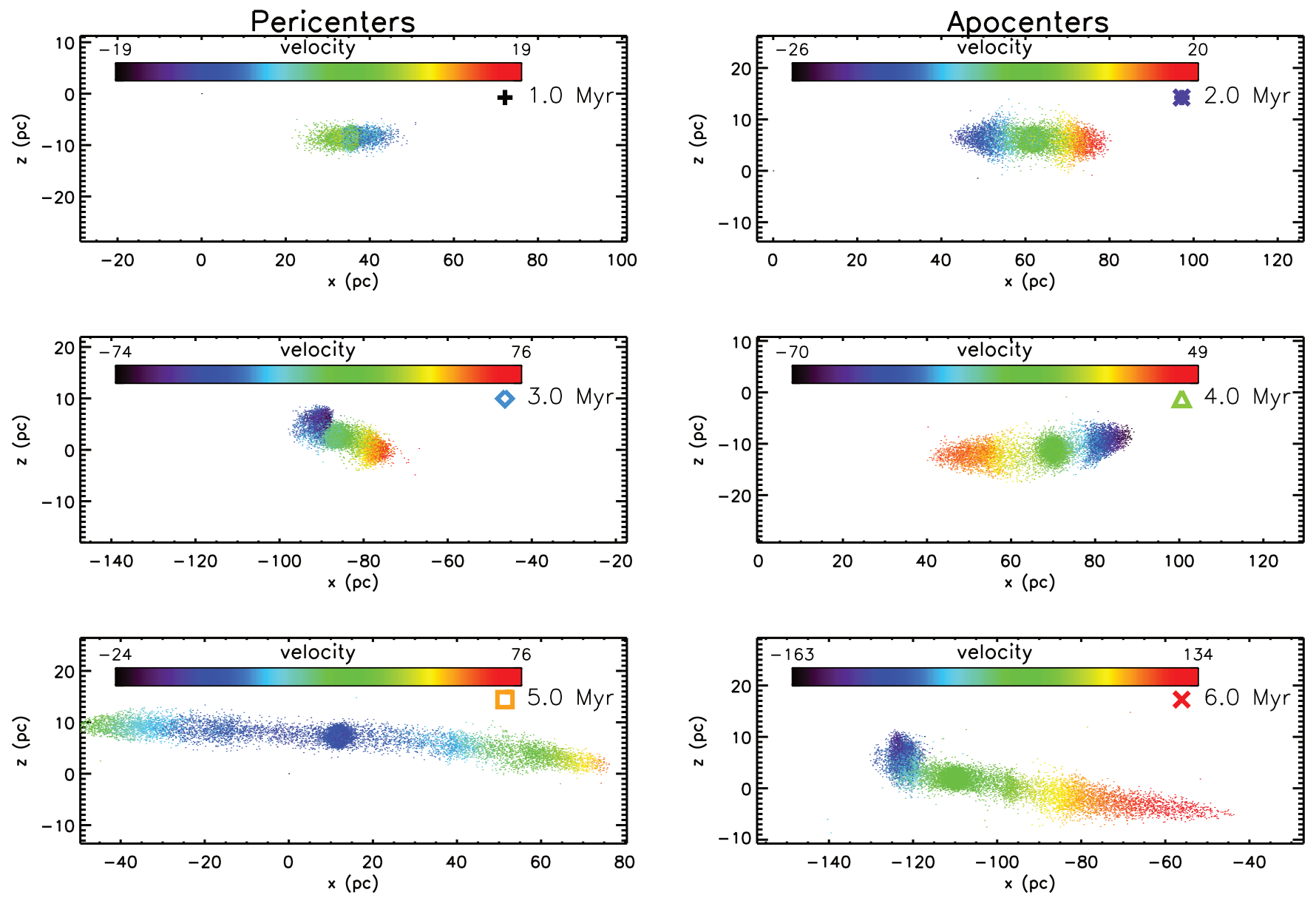

(b)

Fig. A.1. a) Projected orbit of the cluster on the plane of the sky, $x z$-plane, is illustrated. The symbols on the orbit mark the apsides of the 3D orbit. Each symbol/color corresponds to a particular age. The thick symbols display the exact position of the apsides, while the thin symbols refer to the closest available snapshot of the simulated cluster by considering the time resolution of the simulations. b) Projected snapshots of the cluster on the plane of the sky are shown at apocenters and pericenters. Each snapshot corresponds to one marked apside on the projected orbit. The sources are colored based on the present velocity variation in the cluster and its tidal tails.

appears squished on the plane of the sky (see Fig. A.1b), while comparing this snapshot to its projected snapshot on the $x y$-plane (Fig. 1b) reveals that the cluster is physically expanded at this location. The same effect is observed for some of our analyzed models. Figure 9a shows that at the age of 4.5 Myr the cluster is located in the middle of its two apside points; hence, it is physically expanded. However, it appears squished on the plane of the sky. The effect of projection causes the massive stars in the tidal arms of the cluster to spread over a much smaller area on the plane of the sky. Models with $20 M_{\odot}$ and $40 M_{\odot}$ as an initial 
mass of a WR progenitor star produce an adequate number of massive stars in the model (see Table 3), which gives us better statistics, so that we can see this effect reflects in the $H_{\mathrm{d}}$ values of models. For these models, the calculated $H_{\mathrm{d}}$ values is higher age of 4.5 Myr for the Quintuplet cluster (see Table 1).

\section{References}

Ankerst, M., Kriegel, H.-P., \& Seidl, T. 1998, IEEE Transactions on Knowledge and Data Engineering, 10, 996

Aurenhammer, F., \& Klein, R. 2000, Voronoi diagrams. In Handbook of Computational Geometry, Chap. V, eds. J. Sack, \& G. Urrutia (Science Publishing: Elsevier), 201

Berukoff, S. J., \& Hansen, B. M. S. 2006, ApJ, 650, 901

Blaauw, A. 1961, Bull. Astron. Inst. Netherlands, 15, 265

Blum, R. D., Schaerer, D., Pasquali, A., et al. 2001, AJ, 122, 1875

Clarkson, W. I., Ghez, A. M., Morris, M. R., et al. 2012, ApJ, 751, 132

Cotera, A. S., Simpson, J. P., Erickson, E. F., et al. 1999, ApJ, 510, 747

Cutri, R. M., Skrutskie, M. F., van Dyk, S., et al. 2003, VizieR Online Data Catalog: II/246

Dong, H., Wang, Q. D., Cotera, A., et al. 2011a, MNRAS, 417, 114

Dong, H., Wang, Q. D., Cotera, A., et al. 2011b, in The Galactic Center: a Window to the Nuclear Environment of Disk Galaxies, ASP Conf. Proc., 439, 104

Dong, H., Wang, Q. D., \& Morris, M. R. 2012, MNRAS, 425, 884

Eckart, A., Genzel, R., Hofmann, R., Sams, B. J., \& Tacconi-Garman, L. E. 1993, ApJ, 407, L77

Eggleton, P. P., Fitchett, M. J., \& Tout, C. A. 1989, ApJ, 347, 998

Ekström, S., Georgy, C., Eggenberger, P., et al. 2012, A\&A, 537, A146

Eldridge, J. J., \& Tout, C. A. 2004, MNRAS, 353, 87

Espinoza, P., Selman, F. J., \& Melnick, J. 2009, A\&A, 501, 563

Ferrière, K., Gillard, W., \& Jean, P. 2007, A\&A, 467, 611

Figer, D. F. 1995, Ph.D. Thesis, University of California, Los Angeles

Figer, D. F. 2008, in IAU Symp. 250, eds. F. Bresolin, P. A. Crowther, \& J. Puls, 247

Figer, D. F., \& Morris, M. 2002, Modes of Star Formation and the Origin of Field Populations, ASP Conf. Proc., 285, 381

Figer, D. F., Kim, S. S., Morris, M., et al. 1999a, ApJ, 525, 750

Figer, D. F., McLean, I. S., \& Morris, M. 1999b, ApJ, 514, 202

Figer, D. F., Najarro, F., Gilmore, D., et al. 2002, ApJ, 581, 258

Gerhard, O. 2001, ApJ, 546, L39

Ghez, A. M., Salim, S., Weinberg, N. N., et al. 2008, ApJ, 689, 1044

Gies, D. R., \& Bolton, C. T. 1986, ApJS, 61, 419

Gillessen, S., Eisenhauer, F., Fritz, T. K., et al. 2009, ApJ, 707, L114

Gvaramadze, V. V., \& Bomans, D. J. 2008, A\&A, 490, 1071

Habibi, M., Stolte, A., Brandner, W., Hußmann, B., \& Motohara, K. 2013, A\&A, 556, A26

Hafner, J., Sawhney, H., Equitz, W., Flickner, M., \& Niblack, W. 1995, Pattern Analysis and Machine Intelligence, IEEE Trans., 17, 729

Hamann, W.-R., Gräfener, G., \& Liermann, A. 2006, A\&A, 457, 1015

Harfst, S., Portegies Zwart, S., \& Stolte, A. 2010, MNRAS, 409, 628

Hills, J. G. 1988, Nature, 331, 687

Homeier, N. L., Blum, R. D., Pasquali, A., Conti, P. S., \& Damineli, A. 2003, A\&A, 408, 153

Hußmann, B. 2014, Ph.D. Thesis, University of Bonn

Hußmann, B., Stolte, A., Brandner, W., Gennaro, M., \& Liermann, A. 2012, A\&A, 540, A57
Hut, P. 2003, Astrophysical Supercomputing using Particle Simulations, eds. J. Makino, \& P. Hut (ASP), IAU Symp., 208, 331

Kim, S. S., Figer, D. F., Lee, H. M., \& Morris, M. 2000, ApJ, 545, 301

King, I. R. 1966, AJ, 71, 64

Küpper, A. H. W., Kroupa, P., Baumgardt, H., \& Heggie, D. C. 2010, MNRAS, 401, 105

Lada, C. J., \& Lada, E. A. 2003, ARA\&A, 41, 57

Langer, N. 1998, A\&A, 329, 551

Liermann, A., Hamann, W.-R., Oskinova, L. M., Todt, H., \& Butler, K. 2010, A\&A, 524, A82

Liermann, A., Hamann, W.-R., \& Oskinova, L. M. 2012, A\&A, 540, A14

Longmore, S. N., Rathborne, J., Bastian, N., et al. 2012, ApJ, 746, 117

Martins, F., Hillier, D. J., Paumard, T., et al. 2008, A\&A, 478, 219

Mauerhan, J. C., Muno, M. P., \& Morris, M. 2007, ApJ, 662, 574

Mauerhan, J. C., Cotera, A., Dong, H., et al. 2010a, ApJ, 725, 188

Mauerhan, J. C., Muno, M. P., Morris, M. R., Stolovy, S. R., \& Cotera, A. 2010b, ApJ, 710, 706

McMillan, S. L. W. 1996, The Origins, Evolution, and Destinies of Binary Stars in Clusters, eds. E.F. Milone, \& J.-C. Mermilliod, ASP Conf. Ser., 90, 413

Meynet, G., \& Maeder, A. 2003, A\&A, 404, 975

Mezger, P. G., Zylka, R., Philipp, S., \& Launhardt, R. 1999, A\&A, 348, 457

Morris, M., \& Serabyn, E. 1996, ARA\&A, 34, 645

Muno, M. P., Bower, G. C., Burgasser, A. J., et al. 2006, ApJ, 638, 183

Muno, M. P., Bauer, F. E., Baganoff, F. K., et al. 2009, VizieR Online Data Catalog J/ApJS/181/110

Nagayama, T., Nagashima, C., Nakajima, Y., et al. 2003, in SPIE Conf. Ser., 4841, eds. M. Iye, \& A. F. M. Moorwood, 459

Najarro, F., Figer, D. F., Hillier, D. J., \& Kudritzki, R. P. 2004, ApJ, 611, L105

Odenkirchen, M., Grebel, E. K., Rockosi, C. M., et al. 2001, ApJ, 548, L165

Olczak, C., Kaczmarek, T., Harfst, S., Pfalzner, S., \& Portegies Zwart, S. 2012, ApJ, 756, 123

Oskinova, L. M., Steinke, M., Hamann, W.-R., et al. 2013, MNRAS, 436, 3357

Poveda, A., Ruiz, J., \& Allen, C. 1967, Boletin de los Observatorios Tonantzintla y Tacubaya, 4,86

Perets, H. B., Hopman, C., \& Alexander, T. 2007, ApJ, 656, 709

Portegies Zwart, S. F., \& Verbunt, F. 1996, A\&A, 309, 179

Portegies Zwart, S. F., Makino, J., McMillan, S. L. W., \& Hut, P. 2001a, ApJ, 546, L101

Portegies Zwart, S. F., McMillan, S. L. W., Hut, P., \& Makino, J. 2001b, MNRAS, 321, 199

Salpeter, E. E. 1955, ApJ, 121, 161

Sana, H., de Mink, S. E., de Koter, A., et al. 2012, Science, 337, 444

Sander, A., \& Hamann, W.-R. 2012, Proc. Scientific Meeting in Honor of Anthony F. J. Moffat, ASP Conf. Ser., 465, 243

Schödel, R., Merritt, D., \& Eckart, A. 2009, A\&A, 502, 91

Stolte, A., Brandner, W., Grebel, E., Lenzen, R., \& Lagrange, A 2005, ApJ, 628, L113

Stolte, A., Ghez, A. M., Morris, M. R., et al. 2008a, J. Phys. Conf. Ser., 131, 012015

Stolte, A., Ghez, A. M., Morris, M., et al. 2008b, ApJ, 675, 1278

Stolte, A., Morris, M., Ghez, A., et al. 2011, Proc. Stellar Clusters Associations: a RIA Workshop on Gaia (Granada), eds. E. J. Alfaro Navarro, A. T. Gallego Calvente, \& M. R. Zapetero Osorio, 304

Stone, R. C. 1991, AJ, 102, 333

Tout, C. A., Aarseth, S. J., Pols, O. R., \& Eggleton, P. P. 1997, MNRAS, 291, 732

Wang, Q. D., Dong, H., Cotera, A., et al. 2010, MMRAS, 402, 895

Yu, Q., \& Tremaine, S. 2003, ApJ, 599, 1129

Zinnecker, H., \& Yorke, H. W. 2007, ARA\&A, 45, 481 